\title{
INITIAL GUESSES FOR SEQUENCES OF LINEAR SYSTEMS IN A GPU-ACCELERATED INCOMPRESSIBLE FLOW SOLVER *
}

\author{
ANTHONY P. AUSTIN ${ }^{\dagger}$, NOEL CHALMERS ${ }^{\ddagger}$, AND TIM WARBURTON§
}

\begin{abstract}
We consider several methods for generating initial guesses when iteratively solving sequences of linear systems, showing that they can be implemented efficiently in GPU-accelerated PDE solvers, specifically solvers for incompressible flow. We propose new initial guess methods based on stabilized polynomial extrapolation and compare them to the projection method of Fischer [15], showing that they are generally competitive with projection schemes despite requiring only half the storage and performing considerably less data movement and communication. Our implementations of these algorithms are freely available as part of the libParanumal collection of GPU-accelerated flow solvers.
\end{abstract}

Key words. initial guesses, iterative solvers, GPU-acceleration, partial differential equations, incompressible flow, projection, extrapolation, least-squares

AMS subject classifications. $65 \mathrm{~F} 10,65 \mathrm{M} 22$

1. Introduction. Solving a time-dependent partial differential equation (PDE), discretized via the method of lines using an implicit (or partially implicit) timestepping scheme boils down to solving a sequence of linear systems, one (sometimes more) at each time step. For large-scale simulations involving discretizations with millions (or even billions) of degrees of freedom, these systems are often too large even to form explicitly, much less solve directly. Thus, one turns to iterative methods $[18,30]$. In particular, Krylov subspace iterations, which include the popular conjugate gradient (CG) [21] and generalized minimal residual (GMRES) [31] algorithms, have proven successful in this area.

With Krylov methods, the computational expense of solving a linear system is simply the product of the number of iterations required and the cost of each iteration. Used as-is, these methods are prone to slow convergence and stagnation; a good preconditioner is essential for keeping iteration counts manageable. Even though preconditioning raises the cost per iteration, the substantial reduction in iterations it yields typically makes the trade-off favorable.

Another way to reduce the number of iterations is to supply the method with a good initial guess for the solution. Good initial guesses are not available in all contexts, but in a time-dependent PDE simulation, it is natural to use the solution computed at the previous time step as a guess for the solution at the current one. This requires no effort to implement and can be effective if the solution to the PDE varies smoothly in time.

In a 1998 paper, Fischer [15] showed that one can form an even better initial guess by combining the solutions at several previous time steps instead of just one. Specifically, his method takes as the initial guess the solution to the system whose

\footnotetext{
*Submitted to the editors September 25, 2020.

Funding: This research was supported in part by the Exascale Computing Project (17-SC20-SC), a collaborative effort of the U.S. Department of Energy Office of Science and the National Nuclear Security Administration. This research was also supported in part by the John K. Costain Faculty Chair in Science at Virginia Tech. The first author additionally acknowledges support through the Research Initiation Program at the Naval Postgraduate School.

${ }^{\dagger}$ Dept. of Applied Mathematics, Naval Postgraduate School, 833 Dyer Rd., Monterey, CA 93940

$¥$ AMD Research, Advanced Micro Devices Inc., 7171 Southwest Pkwy., Austin, TX 78735

$\S$ Department of Mathematics, Virginia Tech, 225 Stanger St., Blacksburg, VA 24061
} 
right-hand side is the orthogonal projection of the right-hand side of the linear system at the current time step onto the span of the right-hand sides of the systems at a given number of prior time steps. This is equivalent to taking the linear combination of the prior solutions that minimizes the norm of the residual when substituted into the linear system at the current time step. To avoid growth in the storage required as the simulation runs, Fischer periodically restarts the method by discarding the entire history space of stored solutions and right-hand side vectors. In [8], Christensen showed how to avoid this "hard restart" by using a classic technique for updating QR factorizations. This technique was also employed by al Sayed Ali and Sadkane in [3], who compared variants of this projection scheme with initial guess generation methods based on explicit ODE integrators. Fischer's principle application of the projection technique was to incompressible flow, but similar (and in some cases identical) ideas have emerged in other contexts, including nuclear physics [6] and computational electromagnetics [9, 10, 33, 34].

Another method for using prior solutions to generate initial guesses is polynomial extrapolation, i.e., fitting a polynomial curve to the solutions at the previous time steps and forming the initial guess by evaluating the polynomial at the new time step. In the most basic version of this method, the curve is taken to be a simple (Lagrange) interpolant, though approximations based on Taylor expansions [10] have also been proposed. Using the solution at the previous time step is itself a technique of this type - zeroth-order or constant interpolation - and there are many examples in the literature of codes and algorithms that employ first-order (linear) and second-order (quadratic) interpolation to compute initial guesses based on the previous two or three time steps, respectively. Extrapolation based on higher-order polynomial interpolation has also been considered [6, 19, 26,32], but the results are mixed in general, with authors typically pinning any inefficacy on the poor behavior of polynomial interpolation in equi-spaced points. Because of this, it would seem that polynomial extrapolation is inherently limited compared to projection: while the latter can potentially profitably use data from as many previous time steps as one is willing to store, the former is limited to using just a handful of time steps. Moreover, right-hand side projection is guaranteed to provide a reduction in the residual norm with each additional stored datum. As Fischer points out in [15], there is no such guarantee for extrapolation.

This article has two primary purposes. The first is to revisit these initial guess techniques - projection and extrapolation - in the context of PDE solvers running on modern high-performance computing (HPC) architectures that employ graphics processing units (GPUs) as accelerators. GPUs can be challenging to program effectively; naive implementations of algorithms on GPUs are rarely the most efficient. Nevertheless, as GPU-based architectures become increasingly common ${ }^{1}$ the demand for implementations of algorithms that use the GPU hardware efficiently is rising.

The second purpose is to show that the outcome of the competition between projection and extrapolation for producing superior initial guesses is not as clear cut as the arguments made above might suggest. First, we propose a stabilized polynomial extrapolation method based on solving a least-squares problem instead of naive Lagrange interpolation $[12,29]$, an idea that seems to be new within the context of flow solvers,

\footnotetext{
${ }^{1}$ At the time of this writing, the two most powerful HPC systems in the United States-Summit at Oak Ridge National Laboratory and Sierra at Lawrence Livermore National Laboratory - derive the majority of their computing power from GPUs. Moreover, the Aurora, Frontier, and El Capitan supercomputers to be delivered in 2021-2022 as part of the U.S. Department of Energy's Exascale Computing Project will all have GPU-based architectures.
} 
though it has appeared before in the computational chemistry literature [20, 28]. We further propose a sparse polynomial extrapolation method, which computes a Lagrange interpolant through a subset of the retained history data corresponding to time points with a more favorable distribution for polynomial interpolation, analogous to the "mock-Chebyshev" subsampling idea proposed in [5]. We will see that while projection does tend to provide greater reductions in Krylov solver iteration counts compared with either of these extrapolation methods, it does so at a comparatively high cost: the process of extrapolation is considerably cheaper, amounting to taking a single, fixed linear combination of the history data, whereas projection additionally requires orthogonalization among other things. Extrapolation moves considerably less data through the GPU (or CPU), performs fewer floating-point operations, and, unlike projection, does not require any global reductions (e.g., inner products), so it may be carried out in parallel without the need for inter-node communication. Extrapolation also requires approximately half as much storage as projection and is simple to implement, even on a GPU. Our experiments show that these advantages make extrapolation generally competitive with projection, if not slightly superior to it in some circumstances.

We proceed as follows. In Section 2, we review the method of Fischer and the improvement thereto due to Christensen. In Section 3, we introduce our new initial guess schemes based on stabilized and sparse polynomial extrapolation. As our principle application of interest is computational fluid dynamics, we then in Section 5 describe a GPU-accelerated solver for the incompressible Navier-Stokes (INS) equations that we use as a test-bed. In Section 6, we discuss our GPU implementation of these initial guess methods and illustrate their performance within our INS solver. Additionally, we make observations about how the effectiveness of these methods changes with the Reynolds number of the flow being simulated. Both our INS solver and our implementation of the initial guess schemes are distributed with the libParanumal collection of GPU-accelerated flow solvers [7].

2. Fischer's Successive Right-Hand-Side Projection Method. In purely linear algebraic terms, Fischer's method for generating initial guesses via successive right-hand-side projection works as follows. Let $A$ be an invertible square matrix, and suppose that we have found the solutions $x_{1}, \ldots, x_{n}$ to a sequence of linear systems governed by $A$ with corresponding right-hand sides $b_{1}, \ldots, b_{n}$ and that we want to compute the solution $x_{n+1}$ to the system with right-hand side $b_{n+1}$. That is, we have $A x_{1}=b_{1}, A x_{2}=b_{2}, \ldots A x_{n}=b_{n}$, and we want to solve $A x_{n+1}=b_{n+1}$.

The key idea is to consider the orthogonal projection $\hat{b}_{n+1}$ of $b_{n+1}$ onto the span of $b_{1}, \ldots, b_{n}$. Writing $\hat{b}_{n+1}$ as a linear combination of $b_{1}, \ldots b_{n}$,

$$
\hat{b}_{n+1}=\alpha_{1} b_{1}+\cdots+\alpha_{n} b_{n},
$$

we have $A \hat{x}_{n+1}=\hat{b}_{n+1}$, where $\hat{x}_{n+1}$ is the same linear combination of $x_{1}, \ldots, x_{n}$ :

$$
\hat{x}_{n+1}=\alpha_{1} x_{1}+\cdots+\alpha_{n} x_{n}
$$

This choice of $\hat{x}_{n+1}$ is optimal in the sense that it minimizes the residual norm $\| b_{n+1}-$ $A x \|_{2}$ among all linear combinations $x$ of $x_{1}, \ldots, x_{n}$.

If $\hat{b}_{n+1} \approx b_{n+1}$ - that is, if $b_{n+1}$ almost lies in the span of $b_{1}, \ldots, b_{n}$ - then we will have $x_{n+1} \approx \hat{x}_{n+1}$, provided that $A$ is not badly conditioned. Thus, $\hat{x}_{n+1}$ ought to make a good initial guess for a Krylov subspace method applied to $A x_{n+1}=b_{n+1}$. In particular, this is likely to happen when $b_{1}, \ldots, b_{n+1}$ are the right-hand sides for 
linear systems at a group of successive time steps in a PDE simulation, as they are frequently constructed using similar formulae applied to similar inputs and thus tend to resemble one another fairly closely.

This procedure is simple to implement. All one needs is a means for computing the orthogonal projection $\hat{b}_{n+1}$, and this can be accomplished straightforwardly using the Gram-Schmidt process to compute an orthonormal basis for $\operatorname{span}\left\{b_{1}, \ldots, b_{n}\right\}$. Expanding the basis to retain information from the linear systems solved at each new time step is easy: just do one additional step of Gram-Schmidt to orthogonalize the new right-hand side against the existing basis. Some form of reorthogonalization is necessary to guard against loss of orthogonality in finite-precision arithmetic. The standard strategy is to simply do each orthogonalization twice, which is (provably) usually sufficient $[25, \S 6.9]$.

There are two issues that must be addressed. First, when expanding the history space of retained right-hand sides, one must guard against adding vectors to the basis that are too similar to the ones already there (in the sense of near linear dependence). We have observed that failure to do this diminishes the quality of later computed initial guesses and can even result in the simulation becoming unstable. Fortunately, this is easy enough to detect: if the norm of the residual after projection, $\left\|b_{n+1}-\hat{b}_{n+1}\right\|$, is very small relative to $b_{n+1}$, then $b_{n+1}$ already nearly belongs to $\operatorname{span}\left\{b_{1}, \ldots, b_{n}\right\}$, so adding it to the history space is pointless. The threshold for making this determination will depend on the parameters of the PDE solver under consideration; at a minimum, it will depend on the stopping criterion for the linear solver.

Second, in the absence of unlimited storage, it is not possible to continue expanding the space of retained right-hand sides indefinitely: one needs storage for two vectors - one for the right-hand side and one for the corresponding solution - for each time step whose history is to be kept in the space. In [15], Fischer addressed this problem by periodically restarting the technique - discarding the space built to that point and beginning anew - after taking a given fixed number of time steps. We refer to the resulting initial guess generation algorithm as the "classic" variant of Fischer's technique, and pseudocode for it is presented in Algorithm 2.1.

This wholesale discarding of the history space seems wasteful, and it is natural to wonder if there might be a better way. In [8], Christensen proposed dropping a single time step's worth of information from the history space at a time using a standard linear algebra technique for updating Gram-Schmidt QR factorizations [11], [17, §6.5.2]. Once the history space reaches a preselected maximum dimension, Christensen applies the update technique to eliminate the right-hand side corresponding to the oldest retained time step,. Once the linear system is solved, the new right-hand side from the current time step can then be added to the history space in the usual way. Thus, the history space forms a "rolling window" of information from the previous few time steps, and for this reason, we refer to this method as the "rolling QR" variant of this algorithm. Pseudocode may be found in Algorithm 2.2.

The listings in Algorithms 2.1 and 2.2 employ the following notation. The vector $b$ is the new right-hand side for which a solution is sought. The columns of the matrices $\tilde{B}$ and $\tilde{X}$ contain the history space of previous right-hand sides and corresponding solutions, respectively, so that $A \tilde{X}=\tilde{B}$. The columns of $\tilde{B}$ are orthonormal. The variable $d$ is the current dimension of the history spaces, and $M$ is the maximum allowable dimension. The number $\varepsilon$ is a tolerance for determining whether $b$ is too nearly linearly dependent on the columns of $\tilde{B}$ to warrant adding it to the space once the system for it is solved. The vector $x_{0}$ is an arbitrary input for the initial guess when $d=0$. In Algorithm 2.2, $R$ is the triangular factor in the $Q R$ decomposition 


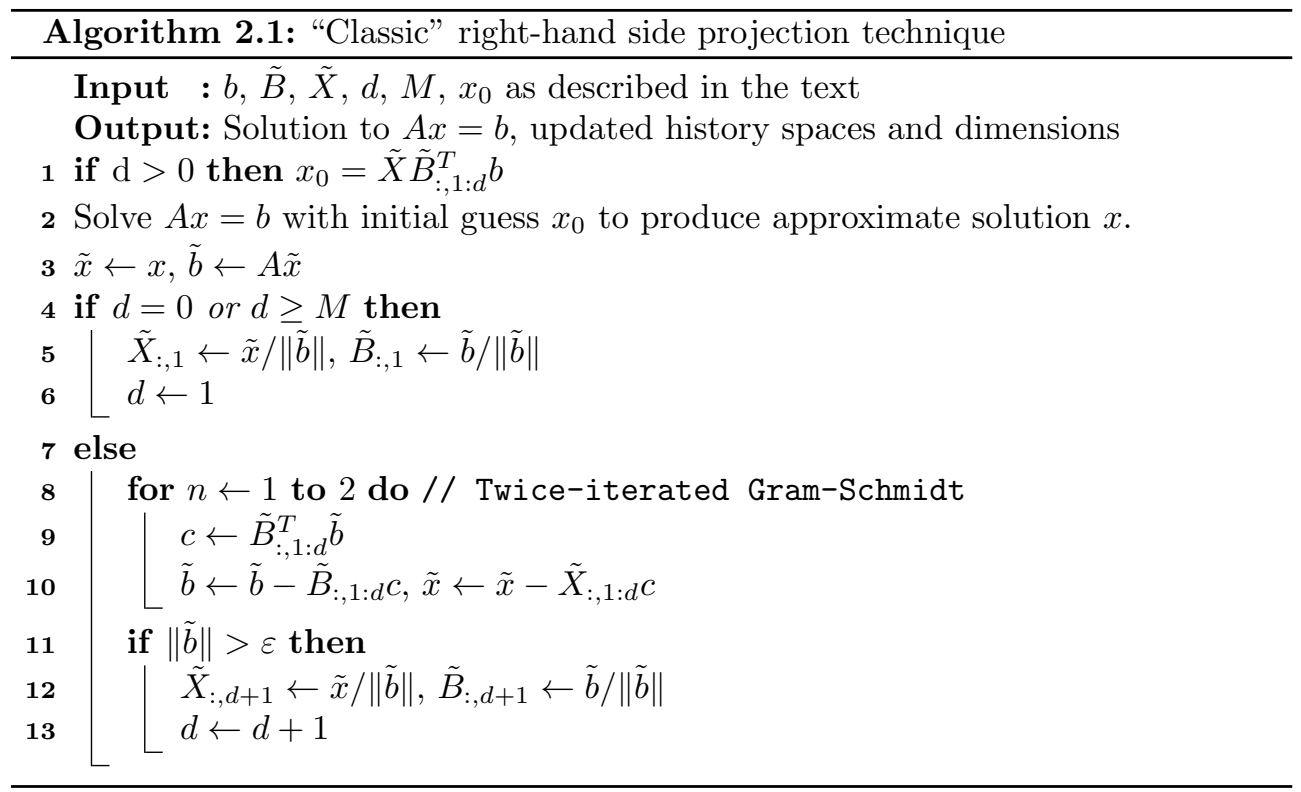

of the right-hand side history space (in which $\tilde{B}$ plays the role of $Q$ ). Matrices are indexed using a MATLAB-like colon notation, e.g., $\tilde{B}_{:, i:(i+1)}$ refers to columns $i$ and $i+1$ of $\tilde{B}$. For any matrix $L$, the symbol $L^{T}$ denotes the transpose of $L$.

3. Stabilized Polynomial Extrapolation Methods. Compared with projection, extrapolation is both an older and more widely-used method for computing initial guesses. Like projection, it forms the guess as a linear combination of prior solutions, but it chooses the coefficients of the combination differently.

In our discussion, we employ the following notation, which differs slightly from that of the previous section: given solutions $x_{n-M+1}, \ldots, x_{n}$ to systems at $M$ previous time steps, we form an estimate $\hat{x}_{n+1}$ for the solution $x_{n+1}$ at the current time step. We denote the coefficients of the linear combination that forms $\hat{x}_{n+1}$ by $\beta_{i}$, writing

$$
\hat{x}_{n+1}=\sum_{i=1}^{M} \beta_{i} x_{n-M+i},
$$

i.e., $\beta_{1}$ multiplies the oldest stored value, $\beta_{2}$ the next oldest, and so forth. To keep the discussion concrete, we focus primarily on the case of a fixed time step, but our remarks apply to variable time step schemes as well with only minor modifications.

3.1. Extrapolation via Lagrange Interpolation. The most common extrapolation technique by far is simple Lagrange polynomial interpolation: compute a polynomial of degree $M-1$ that interpolates $x_{n-M+1}, \ldots, x_{n}$ and determine $\hat{x}_{n+1}$ by evaluating that polynomial at the current time step. Using the solution at the previous time step is one example of this strategy with a degree-0 (constant) interpolating polynomial; this corresponds to the case where $M=1$ and $\beta_{1}=1$. As is widely known, if the size of the time step is constant, the coefficients $\beta_{i}$ have a simple closed-form expression:

TheOREm 3.1. For extrapolation of data given at $M$ equi-spaced points via La- 


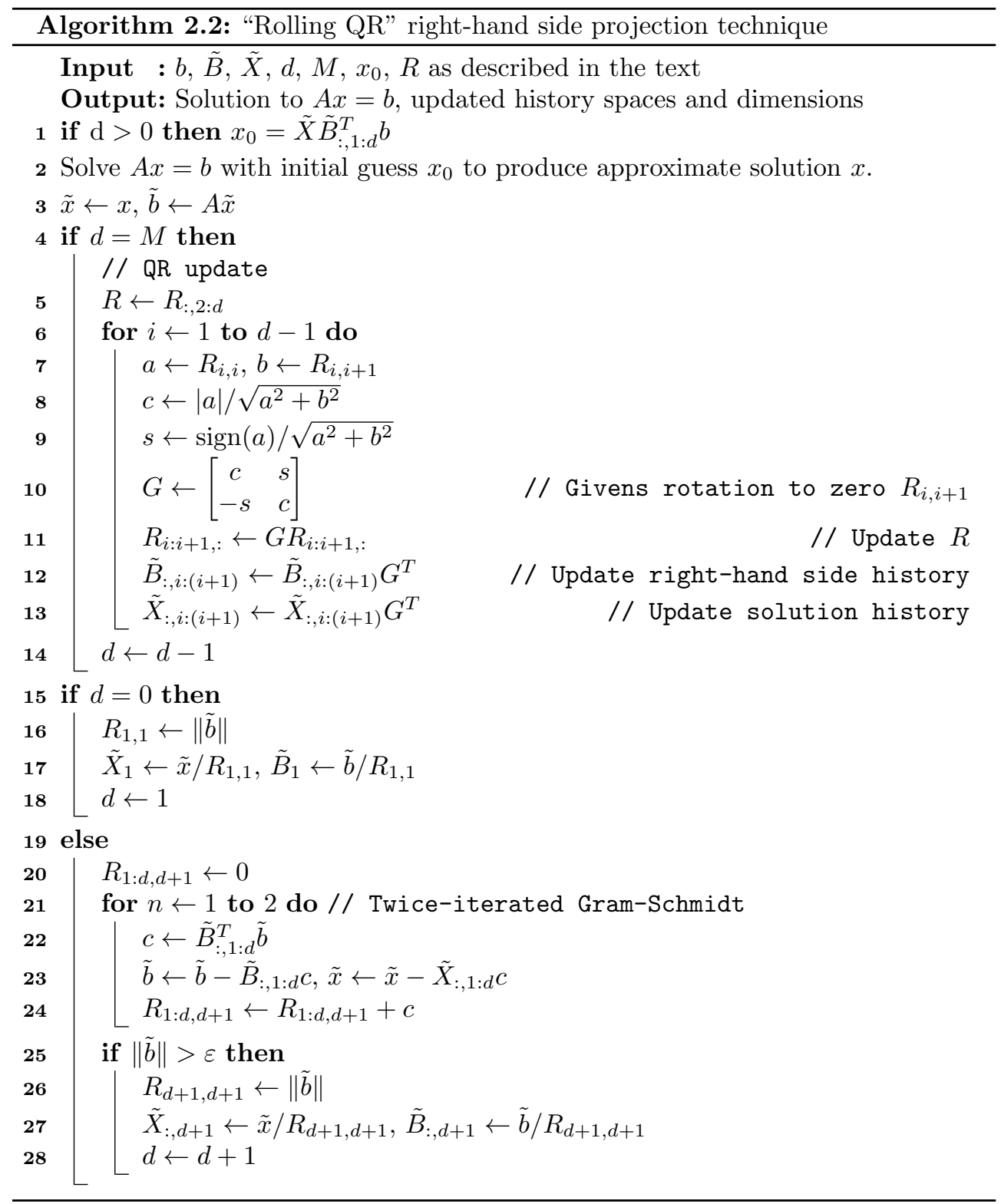

grange interpolation by a polynomial of degree $M-1$,

$$
\beta_{i}=(-1)^{M-i}\left(\begin{array}{c}
M \\
i-1
\end{array}\right) .
$$

The proof, which we omit, is a straightforward application of Newton's forward difference formula [2, 25.2.28], [22] and standard identities involving binomial coefficients. As an example, extrapolation via a linear fit with a fixed time step has $M=2$, $\beta_{1}=-1$, and $\beta_{2}=2$. These coefficients are tabulated in several places in the literature, e.g. [32, Table 1], and, ignoring sign, can be found on the $(M+1)^{\text {th }}$ row of Pascal's triangle. 
This scheme is simple but runs into trouble in practice as $M$ increases. This is typically blamed on the fact that the polynomial interpolation problem can be poorly conditioned if the interpolation points are not chosen appropriately. Equi-spaced interpolation in particular (which corresponds in our context to Lagrange interpolation of solution history with a fixed time step) is known to become exponentially poorly conditioned as the number of points increases and moreover can fail to converge even in theory for well-behaved functions, a property known as the Runge phenomenon [35]. The resulting algorithmic instability limits the utility of extrapolation via Lagrange interpolation to small values of $M$ and low polynomial orders. This would seem to be a severe disadvantage for extrapolation compared to projection methods, which can use as much history data as one is willing to store to further improve the initial guess.

3.2. Stable Polynomial Extrapolation via Least-Squares. We posit that this limitation can be overcome by switching from interpolation to least-squares fitting. While polynomial interpolation at equi-spaced points deserves its poor reputation, recent work $[12,29]$ has shown that polynomial least-squares fitting can be performed stably provided that the number of data points $M$ increases at a rate proportional to the square of the degree $m$ of the polynomial being fit: $M=O\left(m^{2}\right)$ as $m$ becomes large. While this technique has been known to approximation theorists for some time, it seems to have been largely overlooked for use in generating initial guesses within PDE solvers, despite some precedence in [20, 28].

The price paid for this stability is a reduction in the order of accuracy: even when the data to be fit consists of samples of values of a holomorphic function, the rate of convergence of the scheme is only root-exponential in $M$ as $M$ increases, while a wellconstructed interpolation scheme using good points would converge geometrically. This trade-off is inevitable, as it is impossible to construct a stable algorithm for approximating data from equi-spaced samples that converges at a geometric rate [27]. Nevertheless, as our experiments in Section 6 show, the initial guesses generated by this stabilized polynomial extrapolation scheme can be quite effective at reducing solver iteration counts and the time to solution.

Given a polynomial degree $m$ and a history space of size $M \geq m+1$, we can compute the coefficients $\beta_{i}$ as follows. First, we lose no generality in assuming that $x_{n-M+1}, \ldots x_{n}$ are scalar values rather than vectors, as vector data is handled merely by extrapolating each component. Since we assume a fixed time step, we also lose no generality in assuming that the time points at which the history data are provided are the equally spaced points $t_{i}=-1+(i-1) h$ in $[-1,1]$, where $h=2 /(M-1)$ and $1 \leq i \leq$ $M$. For if not, we can make them so by applying an affine transformation, which does not change the solution to the least-squares problem. Under this assumption, the new time point - the point at which the extrapolating polynomial must be evaluated - is $t_{n+1}=1+h$.

Let $\psi_{0}, \ldots \psi_{m}$ be any basis for the space of polynomials of degree at most $m$ that is well-behaved on $[-1,1]$, such as Legendre or Chebyshev polynomials, and consider the Vandermonde-like matrix $V$ whose $(i, j)$ entry is $V_{i j}=\psi_{j}\left(t_{i}\right)$ for $1 \leq$ $i \leq M$ and $0 \leq j \leq m$. Expressing the extrapolation polynomial in this basis as $\psi(t)=c_{0} \psi_{0}(t)+\cdots+c_{m} \psi_{m}(t)$, the overdetermined linear system for the expansion coefficients $c_{0}, \ldots c_{m}$ is

$$
V c=x,
$$


where

$$
c=\left[\begin{array}{c}
c_{0} \\
\vdots \\
c_{m}
\end{array}\right] \quad \text { and } \quad x=\left[\begin{array}{c}
x_{n-M+1} \\
\vdots \\
x_{n}
\end{array}\right]
$$

Noting that $V$ is full-rank, the unique least-squares solution to this system is

$$
c=\left(V^{T} V\right)^{-1} V^{T} x
$$

With $c$ in hand, we evaluate

$$
\hat{x}_{n+1}=\psi\left(t_{n+1}\right)=v^{T} c,
$$

where $v$ is a column vector of length $m+1$ with $j^{\text {th }}$ component $v_{j}=\psi_{j}\left(t_{n+1}\right)$, $0 \leq j \leq m$. Thus,

$$
\hat{x}_{n+1}=v^{T}\left(V^{T} V\right)^{-1} V^{T} x,
$$

from which it follows that

$$
\beta^{T}=v^{T}\left(V^{T} V\right)^{-1} V^{T}
$$

where $\beta^{T}=\left[\begin{array}{lll}\beta_{1} & \cdots & \beta_{M}\end{array}\right]$.

Note that once we have $\beta$ in hand, we no longer need $c$; the expansion (3.1) does not use the least-squares polynomial directly. In particular, we do not need to carry out the fitting procedure just described for each component of the vectors.

3.3. Sparse Polynomial Extrapolation. There is another way to characterize the coefficients $\beta_{1}, \ldots, \beta_{M}$ just computed that turns out to be insightful. Observe that (3.4) implies

$$
\beta^{T} V=v^{T}
$$

To interpret this equation, recall that the $j^{\text {th }}$ column of $V$ consists of the values of $\psi_{j}$ at the prior time points, so the inner product of $\beta$ with this column gives the value obtained by the extrapolation scheme when the history data consist of samples of $\psi_{j}$. Since the $j^{\text {th }}$ element of $v$ is the value of $\psi_{j}$ at the new time point, it follows that the extrapolation scheme is exact in this case. It then follows by linearity that the scheme is exact if the history data consist of samples of any polynomial of degree at most $m$. Of course we do not need (3.5) to tell us this; it follows from the fact that $c$ was chosen to solve (3.3) in a least-squares sense. If $x$ belongs to $\operatorname{Ran}(V)$, then $c$ can be chosen so that the residual vanishes.

But what if, instead of (3.3), we take (3.5) as our starting point and demand only that our extrapolation scheme yield exact results for degree- $m$ polynomial data? The equation (3.5) defines an underdetermined system for the unknown row vector $\beta^{T}$ and thus has infinitely many solutions. The choice of $\beta^{T}$ given by (3.4) has the distinction of being the solution to (3.5) that has minimum norm, a consequence of the fact that the matrix $\left(V^{T} V\right)^{-1} V^{T}$ is the (Moore-Penrose) pseudoinverse of $V$. In essence, this minimality property says that the extrapolation scheme derived from (3.4) is the "most stable" of all that can be derived from (3.5), and in general, this is a good reason to prefer it. Nevertheless, it is natural to ask if there are other solutions to (3.5) that yield extrapolation schemes of interest. 
As a potential alternative to the pseudoinverse solution, we propose to use extrapolation schemes derived from solutions to (3.5) obtained via a column-pivoted QR factorization $[17, \S 5.6 .2]$. First, observe that (3.5) is equivalent to

$$
V^{T} \beta=v \text {. }
$$

Write $V^{T} P=Q R$, where $Q$ is an $(m+1) \times(m+1)$ orthogonal matrix, $R$ is an $(m+1) \times M$ upper trapezoidal matrix, and $P$ is an $M \times M$ permutation matrix chosen by the column pivoting. Then, since $P^{-1}=P^{T}$, we have

$$
Q R P^{T} \beta=v \text {. }
$$

Now, partition $R$ and $P^{T} \beta$ as

$$
R=\left[\begin{array}{ll}
\hat{R} & \tilde{R}
\end{array}\right] \quad \text { and } \quad P^{T} \beta=\left[\begin{array}{c}
\hat{\beta} \\
\tilde{\beta}
\end{array}\right]
$$

where $\hat{R}$ is $(m+1) \times(m+1), \tilde{R}$ is $(m+1) \times(M-m-1)$ and $\hat{\beta}$ and $\tilde{\beta}$ are length $m+1$ and $M$, respectively. Then, we have

$$
\hat{R} \hat{\beta}+\tilde{R} \tilde{\beta}=Q^{T} v .
$$

and we are free to choose $\hat{\beta}$ and $\tilde{\beta}$ to make this equation hold. One natural choice is to set $\tilde{\beta}=0$. Then, since $V$ is full-rank, $\hat{R}$ is invertible, and we have $\hat{\beta}=\hat{R}^{-1} Q^{T} v$. It follows that

$$
\beta=P\left[\begin{array}{c}
\hat{R}^{-1} Q^{T} v \\
0
\end{array}\right]
$$

is another choice for $\beta$ that satisfies (3.5).

The advantage of choosing $\beta$ according to (3.6) instead of (3.4) is that with the former, only $m+1$ of the entries are nonzero, which reduces the amount of computational effort required to evaluate (3.1). For this reason, we refer to this scheme as sparse polynomial extrapolation. The sacrifice made is a little bit of stability: in general, (3.6) is not the minimum-norm solution to (3.5), so extrapolation with (3.6) will have a greater propensity to "amplify" the history data. Nevertheless, the column pivoting ensures that this loss of stability is minimal, and we will see in Section 6.3 that both (3.3) and (3.6) lead to successful extrapolation schemes in practice.

Another way to view this scheme is as follows. Since only $m+1$ of the coefficients are nonzero, yet (3.6) solves the exactness equation (3.5) for polynomials, it follows that an extrapolation scheme based on (3.6) is actually computing a degree- $m$ polynomial interpolant through the data points corresponding to the nonzero coefficients. What sets this interpolation scheme apart from the basic one discussed in Section 3.1 is which points are chosen. The basic scheme simply uses the last $m+1$ data points, while the scheme based on (3.6) adaptively selects $m+1$ of the previous $M$ data points to yield a scheme with better stability properties.

This idea is not without precedent in the literature. Bos and coauthors used column-pivoted QR in [4] to select good sets of points for multivariate interpolation. In [5], Boyd and Xu proposed what they call "mock-Chebyshev subset interpolation", in which they select $m+1$ interpolation points from an equi-spaced grid of $O\left(m^{2}\right)$ points so that they are distributed similarly to Chebyshev points, which are a near-optimal set of points for polynomial interpolation [35]. The points selected by the columnpivoted QR procedure exhibit similar end point clustering to Chebyshev points. 
4. Comparison of Computational Costs. It would seem at first glance that the minimality property satisfied by the projection schemes makes them obviously superior. In more detail, we have the following result:

Theorem 4.1. Consider a sequence $A x_{n-M+1}=b_{n-M+1}, \ldots, A x_{n}=b_{n}$ of $M$ linear systems, where $x_{i}=x(i h)$ and $b_{i}=b(i h)$ are equally-spaced samples of smooth functions $x(t)$ and $b(t)$ for some $h>0$. Let $\hat{x}_{n+1}^{P}$ denote the approximation to $x_{n+1}=$ $A^{-1} b_{n+1}$ obtained using right-hand side projection onto $\operatorname{span}\left\{b_{n-M+1}, \ldots, b_{M}\right\}$, and let $\hat{x}_{n+1}^{E}$ denote the approximation to $x_{n+1}$ obtained by degree-m least-squares extrapolation of $\operatorname{span}\left\{x_{n-M+1}, \ldots, x_{M}\right\}$, where $M \geq m+1$. The residual norms satisfy:

(i) $\left\|b_{n+1}-A \hat{x}_{n+1}^{P}\right\|_{2} \leq\left\|b_{n+1}-A \hat{x}_{n+1}^{E}\right\|_{2}$.

(ii) $\left\|b_{n+1}-A \hat{x}_{n+1}^{P}\right\|_{2}=O\left(h^{M}\right)$ as $h \rightarrow 0$.

(iii) $\left\|b_{n+1}-A \hat{x}_{n+1}^{E}\right\|_{2}=O\left(h^{m+1}\right)$ as $h \rightarrow 0$.

Proof. Statement (i) follows from the fact that $\hat{x}_{n+1}^{P}$ is chosen to minimize $\| b_{n+1}-$ $A x \|_{2}$ over all possible linear combinations $x$ of $x_{n-M+1}, \ldots x_{n}$, and $\hat{x}_{n+1}^{E}$ is one such combination. Statement (ii), proved in [3, Theorem 2.3], is a consequence of the fact that another such combination is given by degree- $(M-1)$ polynomial interpolation, and this can be shown to be $O\left(h^{M}\right)$-accurate using Taylor's theorem.

Statement (iii) can be proved by similar arguments. If $\hat{x}_{n+1}^{I}$ is the initial guess obtained by degree- $m$ polynomial interpolation in $x_{n-m}, \ldots, x_{n}$, then

$$
\left\|x_{n+1}-\hat{x}_{n+1}^{E}\right\|_{2} \leq\left\|x_{n+1}-\hat{x}_{n+1}^{I}\right\|_{2},
$$

since $\hat{x}_{n+1}^{I}$ is a competitor in the least-squares problem $\hat{x}_{n+1}^{E}$ was chosen to solve. Thus,

$$
\left\|b_{n+1}-A \hat{x}_{n+1}^{E}\right\|_{2} \leq\|A\|_{2}\left\|x_{n+1}-\hat{x}_{n+1}^{E}\right\|_{2} \leq\|A\|_{2}\left\|x_{n+1}-\hat{x}_{n+1}^{I}\right\|_{2}=O\left(h^{m+1}\right)
$$

by the result on the accuracy of polynomial interpolation just mentioned.

We will see in Section 6 that the advantage of projection predicted by this theorem is real: initial guesses derived from projection do tend to yield smaller residuals and correspondingly lower solver iteration counts. But this advantage comes at a price. Projection is more expensive to carry out than extrapolation, and if the difference in cost is large enough, the latter may be able to compete when effectiveness is measured in time to solution.

The core operations involved in both projection and extrapolation are easily seen to be memory-bound, and we can therefore compare the cost of the two by counting the number of memory operations that our implementations of these methods perform at each time step. Table 1 shows these counts to leading order for each of the methods we have been considering. In the first column, we introduce some nomenclature for the methods that we will use throughout the remainder of the article. "LAST" refers to the initial guess strategy of using the solution at the previous time step. "CLASSIC $(M)$ " and "QR $(M)$ " refer to the classic and rolling-QR variants of the projection methods with a size- $M$ history space. "EXTRAP $(m, M)$ " refers to leastsquares polynomial extrapolation with degree $m$ over a history space of size $M$, and "SPEXTRAP $(m, M)$ " refers to the sparse variant. The symbol $N_{\text {total }}$ is the dimension of the linear systems. By "leading order", we mean that we have assumed $N_{\text {total }} \gg M$. and therefore can neglect terms in the counts that do not contain $N_{\text {total }}$ as a factor.

Details of the computations for the entries in this table may be found in Appendix A. The point is that extrapolation moves considerably less data than the projection methods. Asymptotically as $M$ becomes large, $\operatorname{EXTRAP}(m, M)$ moves 4 times less 


\begin{tabular}{cccc}
\hline Method & Form guess & Update history space & Total \\
\hline LAST & 0 & 0 & 0 \\
CLASSIC $(M)$ & $(M+3) N_{\text {total }}$ & $(3 M+27) N_{\text {total }}$ & $(4 M+30) N_{\text {total }}$ \\
QR $(M)$ & $2(M+1) N_{\text {total }}$ & $(10 M+24) N_{\text {total }}$ & $(12 M+26) N_{\text {total }}$ \\
EXTRAP $(m, M)$ & $(M+1) N_{\text {total }}$ & 0 & $(M+1) N_{\text {total }}$ \\
SPEXTRAP $(m, M)$ & $(m+2) N_{\text {total }}$ & 0 & $(m+2) N_{\text {total }}$ \\
\hline
\end{tabular}

Table 1: Leading-order counts of memory operations (number of floating-point values loaded and stored) performed per time step in our implementations of each of the initial guess methods. Here, $M$ refers to the size of the history space, $m$ is the polynomial degree used in extrapolation, and $N_{\text {total }}$ is the dimension of the linear systems. By "leading-order", we mean that we assume $N_{\text {total }} \gg M$. The count for $\operatorname{CLASSIC}(M)$ is an average over one restart cycle. Counts for the other methods assume a full history space.

data per time step than $\operatorname{CLASSIC}(M)$ and 12 times less data than $\mathrm{QR}(M)$. For smaller $M$, the factors will be even larger. Sparse extrapolation moves even less data, depending on the choice of polynomial degree.

In addition to moving less data and being simple to implement, extrapolation has two other advantages that make it appealing. First, it requires approximately half the storage of the projection methods, as it needs only a history space of solutions while the projection methods require a history space of right-hand sides as well. Second, the extrapolation methods are entirely local in the sense that they do not require any communication to carry out in parallel. In particular, they do not require any global inner products or global operator evaluations. Projection methods, on the other hand, require both: an operator evaluation to find the right-hand side corresponding to the approximate solution (see line 3 of Algorithm 2.1) and inner products both during orthogonalization and when computing the projections.

5. A GPU-Accelerated Incompressible Navier-Stokes Solver. Our goal in this article is to implement these methods in a GPU-accelerated PDE solver in such a way that they use the GPU hardware efficiently. We consider specifically a solver for the incompressible Navier-Stokes (INS) equations,

$$
\begin{aligned}
\frac{\partial \mathbf{u}}{\partial t}-\nu \nabla^{2} \mathbf{u}+\mathbf{u} \cdot \nabla \mathbf{u}+\nabla p & =0 \\
\nabla \cdot \mathbf{u} & =0,
\end{aligned}
$$

posed in a closed domain $\Omega$ in $\mathbb{R}^{3}$ Here, $\mathbf{u}=\left(u_{x}, u_{y}, u_{z}\right)$ is the velocity field $p$ is the pressure, and $\nu$ is the kinematic viscosity. We assume that $\partial \Omega$ may be written as $\partial \Omega_{D} \cup \partial \Omega_{N}$, where $\partial \Omega_{D}$ and $\partial \Omega_{N}$ are disjoint subsets of $\partial \Omega$ on which we impose the boundary conditions

$$
\begin{array}{rlrl}
\mathbf{u} & =\mathbf{g} & & \text { on } \Omega_{D} \\
\nu \nabla \mathbf{u} \cdot \hat{\mathbf{n}}=p \hat{\mathbf{n}} & & \text { on } \Omega_{N},
\end{array}
$$


respectively, where $\hat{\mathbf{n}}$ denotes the outward-pointing unit normal. Standard manipulations lead to the variational formulation

$$
\begin{array}{r}
\frac{\partial}{\partial t} \int_{\Omega} \mathbf{u} \cdot \mathbf{v} d V+\nu \int_{\Omega} \nabla \mathbf{u}: \nabla \mathbf{v} d V+\int_{\Omega} \mathbf{u} \cdot \nabla \mathbf{u} d V-\int_{\Omega} p(\nabla \cdot \mathbf{v}) d V=0 \\
\int_{\Omega}(\nabla \cdot \mathbf{u}) q=0,
\end{array}
$$

to be satisfied by the solution $\mathbf{u}$ for all test functions $\mathbf{v}, q$ of a suitable regularity, where $\mathbf{v}$ vanishes on $\partial \Omega_{D}$.

The solver we use is a continuous Galerkin variant of the high-order discontinuous Galerkin INS solver described in [23] and is freely available as part of the libParanumal suite of flow solvers [7]. For completeness, we describe the discretization and the salient characteristics of the solver here. Note that while we have described (and will continue to describe) everything in terms of flow problems in three spatial dimensions, a two-dimensional solver may be constructed following a similar prescription.

5.1. Spatial Discretization. We partition $\Omega$ into conforming, nonoverlapping elements, which we take in this article to be hexahedra, though other shapes are also viable. We map each element to the reference cube $[-1,1]^{3}$, on which we define a space of multivariate polynomials consisting of tensor products of univariate polynomials of degree at most $N$ in each spatial variable. We represent polynomials in this space by their values on a tensor product grid of Gauss-Legendre-Lobatto (GLL) nodes, and we seek a piecewise continuous approximation to the solution of (5.1) in $\Omega$ formed by linear combinations of these polynomials on each element.

Thus, on element $e$, we expand the local velocity $\mathbf{u}^{e}$ and pressure $p^{e}$ as

$$
\mathbf{u}^{e}(\mathbf{x})=\sum_{i=1}^{N_{p}} \mathbf{u}_{i}^{e} \ell_{i}(\mathbf{x}) \quad \text { and } \quad p^{e}(\mathbf{x})=\sum_{i=1}^{N_{p}} p_{i}^{e} \ell_{i}(\mathbf{x}),
$$

where $N_{p}=(N+1)^{3}$ is the dimension of the local polynomial space (i.e., the number of tensor product GLL nodes) and $\ell_{i}$ is the $i^{\text {th }}$ basis function (a tensor product of Lagrange basis functions for the GLL nodes in one dimension). Inserting these expansions into the variational form (5.2) and imposing the Galerkin condition, we arrive the local semidiscrete system

$$
\begin{aligned}
\frac{d \mathbf{u}^{e}}{d t}+\nu \mathbf{L}^{e} \mathbf{u}^{e}+\mathbf{N}^{e}\left(\mathbf{u}^{e}\right)-\mathbf{G}^{e} p^{e} & =0 \\
D^{e} \mathbf{u}^{e} & =0,
\end{aligned}
$$

where we abuse notation and identify $\mathbf{u}^{e}$ and $p^{e}$ with vectors of the expansion coefficients $\mathbf{u}_{i}^{e}$ and $p_{i}^{e}$, and where $\mathbf{L}^{e}, \mathbf{N}^{e}, \mathbf{G}^{e}$, and $D^{e}$ are the discrete (vector) Laplacian, advection, gradient, and divergence operators, respectively.

Demanding that the solution be continuous across across elements, we assemble the element-local semidiscrete systems into a global semidiscrete system

$$
\begin{aligned}
\frac{d \mathbf{u}}{d t}+\nu \mathbf{L u}+\mathbf{N}(\mathbf{u})-\mathbf{G} p & =0 \\
D \mathbf{u} & =0
\end{aligned}
$$

where we again abuse notation, identifying $\mathbf{u}$ and $p$ with vectors of expansion coefficients of the approximate solution in the global piecewise polynomial basis. That is, 
they are vectors of the values of the computed solution at the union over all elements of the mapped tensor product GLL grids with redundant points shared by multiple elements on their corners, edges, and faces eliminated. The operators $\mathbf{L}, \mathbf{N}, \mathbf{G}$, and $D$ are assembled from the local operators $\mathbf{L}^{e}, \mathbf{N}^{e}, \mathbf{G}^{e}$, and $D^{e}$ in the usual way.

Our implementation is "matrix-free": we do not explicitly form the global matrices $\mathbf{L}, \mathbf{G}$, and $D$, nor do we work directly with the nonlinear global advection operator $\mathbf{N}$. Instead, we effect the action of these matrices on vectors by applying the local operators to vectors of the local solution values on each element and then enforcing continuity by applying a gather-scatter operation [13, §4.5.1], for which we use the gslib software library $[1,14,36]$. The local operators $\mathbf{L}^{e}, \mathbf{G}^{e}$, and $D^{e}$ are applied using quadrature on the tensor product GLL grid on element $e$. To combat aliasing due to nonlinearity when evaluating the local advection operator $\mathbf{N}^{e}$, we first interpolate the solution to a tensor-product grid of Gauss-Legendre (not Lobatto) quadrature points of size $(N+2)^{3}$ and evaluate the integral with Gauss quadrature.

5.2. Temporal Discretization. We discretize (5.3) in time in an implicitexplicit fashion, using a $3^{\text {rd }}$-order backward differentiation formula to treat the diffusive $\nu L \mathbf{u}$ term and an explicit $3^{\text {rd }}$-order polynomial extrapolation scheme for the nonlinear advective term $\mathbf{N}(\mathbf{u})$. If $\Delta t$ is the time step size, and if $u^{n}$ and $p^{n}$ denote the approximations to $\mathbf{u}$ and $p$ at the $n^{\text {th }}$ time step, we obtain a system of the form

$$
\begin{aligned}
(\gamma I+\nu(\Delta t) \mathbf{L}) \mathbf{u}^{n+1}-(\Delta t) \mathbf{G} p^{n+1} & =\sum_{i=0}^{2} \beta_{i} \mathbf{u}^{n-i}-(\Delta t) \sum_{i=0}^{2} \alpha_{i} \mathbf{N}\left(\mathbf{u}^{n-i}\right) \\
D \mathbf{u}^{n+1} & =0
\end{aligned}
$$

for $\mathbf{u}^{n+1}$ and $p^{n+1}$, given the solution at the previous time steps. As these schemes are not self-starting, we initialize them using similar schemes of lower-order. The coefficients for the schemes may be found in [24, Table 5.2].

Solving the fully-coupled system (5.4) for $\mathbf{u}^{n+1}$ and $p^{n+1}$ simultaneously can be expensive. Instead, we use an algebraic splitting scheme to decouple the solve for $\mathbf{u}^{n+1}$ from that for $p^{n+1}$. First, we evaluate the part of the right-hand side that is known explicitly:

$$
\mathbf{f}=\sum_{i=0}^{2} \beta_{i} \mathbf{u}^{n-i}-(\Delta t) \sum_{i=0}^{2} \alpha_{i} \mathbf{N}\left(\mathbf{u}^{n-i}\right) .
$$

Next, we obtain an initial approximation $\hat{\mathbf{u}}$ to $\mathbf{u}^{n+1}$ by solving the first equation in (5.4) assuming that $p^{n+1}=0$ :

$$
\left(\mathbf{L}+\frac{\gamma}{\nu(\Delta t)} I\right) \hat{\mathbf{u}}=\frac{1}{\nu(\Delta t)} \mathbf{f} .
$$

This approximation does not satisfy the incompressibility constraint. We rectify this by taking $p^{n+1}$ to solve

$$
L p^{n+1}=-\frac{\gamma}{\Delta t} D \hat{\mathbf{u}}
$$

(here, $L$ is the matrix that discretizes the scalar Laplacian) and then assigning

$$
\mathbf{u}^{n+1}=\hat{\mathbf{u}}-\frac{\Delta t}{\gamma} \mathbf{G} p^{n+1}
$$




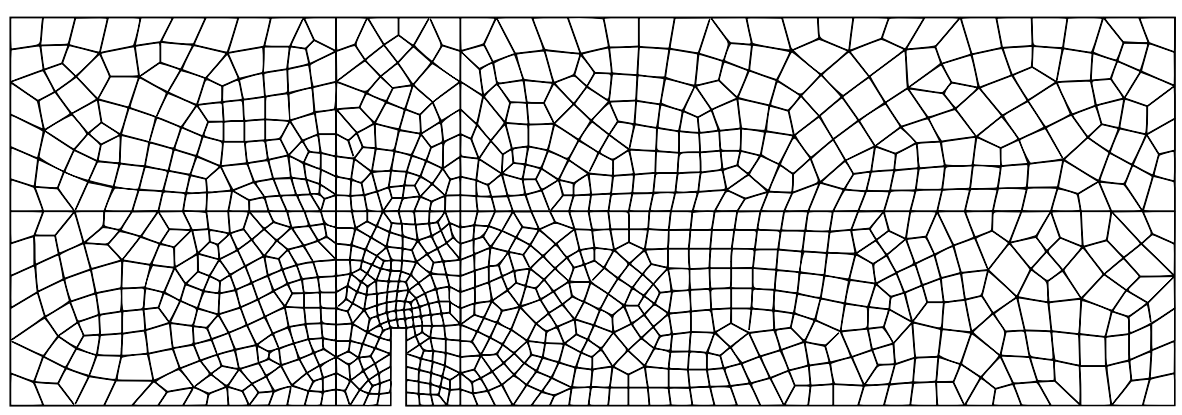

Fig. 1: Two-dimensional cross section of the finite element mesh for 3D channel flow past a rectangular "fence."

The size of the time step for this scheme is limited by the Courant-FriedrichsLewy (CFL) condition associated with the advective term $\mathbf{N}(\mathbf{u}$ ) (since we have treated this term explicitly). We use a semi-Lagrangian subcycling scheme to alleviate this restriction. For further details, see [23, §2.3]

5.3. Linear Solvers and Preconditioning. We solve the elliptic systems (5.5) and (5.6) at each time step using the preconditioned conjugate gradient (PCG) method. We precondition the velocity system (5.5) using a (point) Jacobi approach, which is inexpensive and works well at high Reynolds numbers ( $\operatorname{small} \nu$ ).

For the pressure system (5.6), we use a hybrid $p$-multigrid / algebraic multigrid ( $p \mathrm{MG} / \mathrm{AMG}$ ) preconditioner in which we coarsen the problem (in space) first by using the same discretization but with successively lower polynomial degrees. If coarsening to degree 1 yields a problem that is still too large to solve directly, we further coarsen the problem using an unsmoothed-aggregation algebraic multigrid scheme. We use Chebyshev-accelerated damped-Jacobi smoothing at each level of the multigrid hierarchy. One application of the preconditioner consists of a single V-cycle. For more information, see [16, Ch. 5] and [23].

\section{Numerical Results and Performance.}

6.1. Test Problem and Hardware. We test the effectiveness of using righthand side projection techniques within the solver just described by using them to simulate 3D channel flow past a rectangular "fence" obstacle. The mesh is composed of 8,528 hexahedral elements; a 2D cross-section is displayed in Figure 1. The velocity field is required to satisfy zero Dirichlet conditions on the walls of the channel and along the fence. At the entrance of the channel, we prescribe a uniform rightward inflow condition. At outflow, we use a natural boundary condition.

We perform all of our simulations on either a computer workstation equipped with two NVIDIA TITAN-V GPUs or on a single node of the Cascades cluster at the Advanced Research Computing facility at Virginia Tech. One Cascades node is equipped with two NVIDIA V100 GPUs. Though we have multiple GPUs available in both environments, for simplicity, all of our tests are confined to a single GPU.

6.2. GPU Efficiency. We have implemented both the "classic" and "rolling QR" right-hand-side projection techniques described in Section 2 as well as the extrapolation methods of Section 3 in the INS solver described in the previous section. We maintain separate history spaces of right-hand sides for each of the three com- 
ponents of the velocity and for the pressure. The dimensions of each space may be selected independently from one another, though we do not take advantage of this flexibility in any of our experiments.

The projection methods require four key operations for which we have written GPU kernels: ${ }^{2}$

- rhsProject computes the inner products required to project the right-hand side onto the existing basis, as is done in lines 1 and 9 of Algorithm 2.1. By blocking the inner products together, it reduces data movement compared to the naive algorithm of computing the inner products individually. The inner products are computed using a pointwise vector multiply followed by a standard binary reduction tree with an atomic add at the end.

- rhsReconstruct adds or subtracts linear combinations of vectors in the history space from a given, fixed vector. This operation is needed in lines 1 (with the fixed vector equal to zero) and 10 of Algorithm 2.1.

- rhsUpdateSpace performs the operations on line 12 of Algorithm 2.1, which store new vectors in the history space. Combining these operations, including the scalings by $\|\tilde{b}\|$, into one kernel reduces data movement compared with performing the scalings separately (e.g., using basic linear algebra routines) and then copying the vectors into the spaces.

- rhsQRUpdate performs the QR update step in lines 5-13 of Algorithm 2.2.

The extrapolation methods, being considerably simpler, require just a single kernel that computes the required linear combination of the history vectors; we call this kernel extrapKernel.

As mentioned above, these operations are memory-bound, and our kernels are therefore best judged by the bandwidth they attain. To test their performance, we run 48 time steps of our 3D fence simulation on a single TITAN-V GPU using polynomial degrees $N=1,2, \ldots 6$ and using both the classic and rolling QR variants of the projection methods as well as the extrapolation methods with degree $m=2,3,4$. We vary the maximum size $M$ of the history space, trying $M=4,8,12$. On each run, we collect statistics about the kernels' performance using NVIDIA's nvprof profiling utility.

These statistics are displayed in the roofline plots of Figure 2, which shows the average bandwidth attained by the kernels under each configuration as a function of the amount of data they move. We obtained the roofline itself by measuring the bandwidth attained on a large copy operation, which we found to be approximately $580 \mathrm{~GB} / \mathrm{s}^{3}$ The plots indicate that our kernels all operate close to the streaming performance limit.

6.3. Solver Iterations and Simulation Time. Computing initial guesses using either projection or extrapolation instead of just using the solution at the previous time step generally results in substantial reductions in both solver iterations (numbers of PCG steps) and time to solution. In fact, profiling reveals that, on average and for "reasonable" choices of the history space dimension, computing the projection and forming the initial guess with the rolling QR method takes approximately the same amount of time as one PCG iteration (including application of our hybrid multigrid preconditioner), so it will be a net win in terms of time if it saves just two iterations

\footnotetext{
${ }^{2}$ Our descriptions for the first three kernels reference lines in Algorithm 2.1. Of course, they are used in Algorithm 2.2 too.

${ }^{3}$ The datasheet for the TITAN-V indicates a theoretical peak bandwidth of approximately 653 $\mathrm{GB} / \mathrm{s}$.
} 

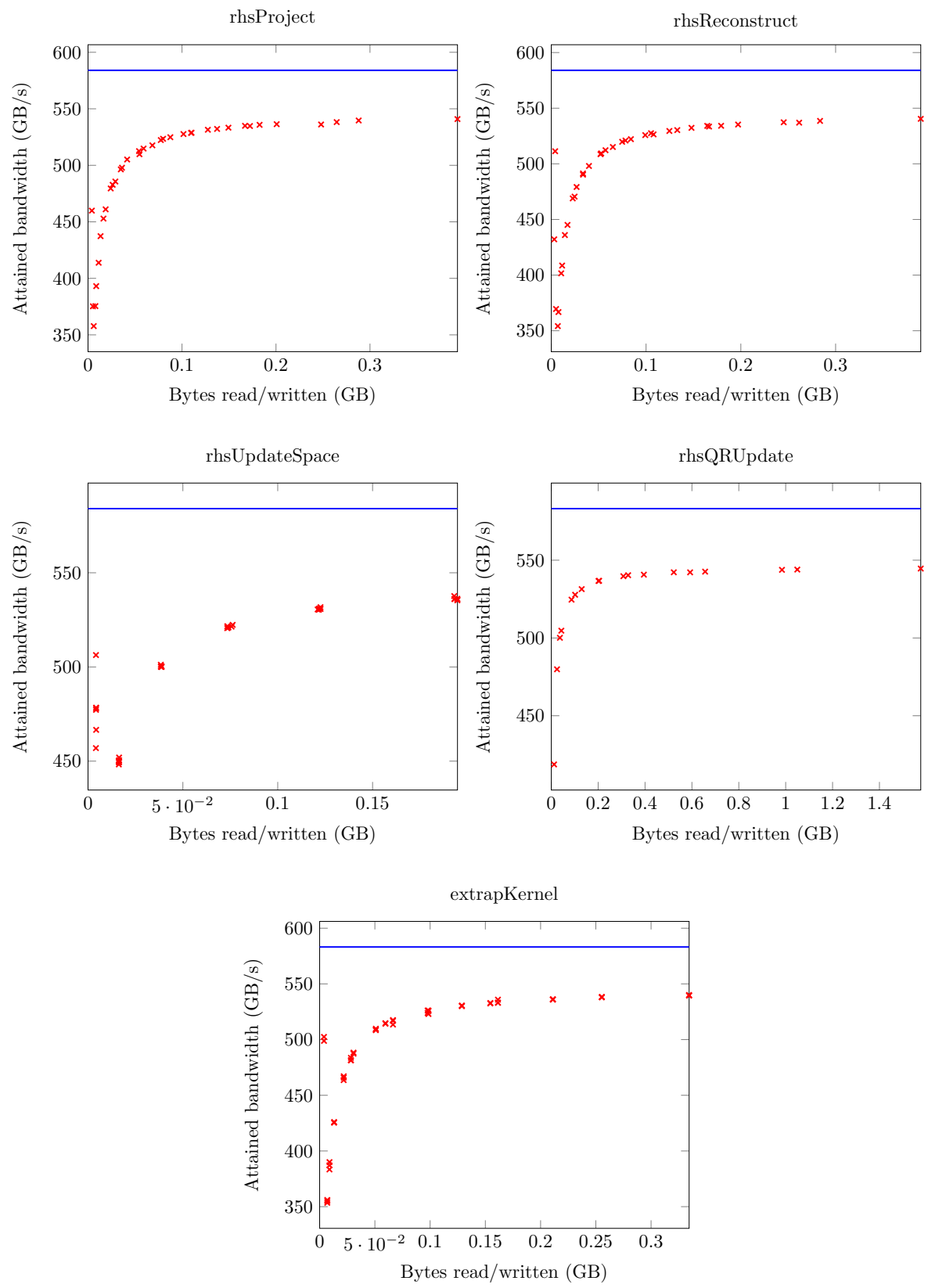

Fig. 2: Roofline plots showing the bandwidth attained by each of the four GPU kernels described in Section 6.2 used for implementing the projection-based initial guess methods. The red crosses indicate the bandwidth attained by the kernel when the size of the input results in the given number of memory transactions. The blue line is an estimate of the maximum attainable memory bandwidth, measured by timing a copy operation on a large array. 
per solve. Extrapolation is approximately ten times faster still.

In general, these methods save considerably more than just two iterations. Table 2 shows the time (in seconds) to run our solver on the 3D fence flow test problem described above to final time $t=1.00$ (8737 time steps) on a single NVIDIA V100 GPU. We ran the solver for flows with Reynolds numbers $\mathrm{Re}=200,500,1000$ (generated by altering the value of the viscosity $\nu$ ) and using nine different initial guess strategies: the solution at the previous time step, the classic projection method, the rolling QR projection method, and both the least-squares and sparse extrapolation methods with degrees $m=2,3,4$. For the projection and extrapolation methods, we used history space dimensions $M=4,8,12$. The numbers in parentheses indicate the speedup obtained with the projection methods relative to using the solution at the last time step.

Even with a history space of dimension just $M=4$ and the classic projection scheme with its naive periodic restart procedure, we obtain a $35-40 \%$ speedup over using the previous time step's solution as an initial guess. Switching to the rolling QR scheme improves this to about $55-60 \%$. While its implementation is more complicated, the rolling QR scheme is clearly superior to the classic one: the classic scheme requires a history space of dimension $M=12$ to attain speedups that the rolling QR scheme is able to attain with $M=4$. We note that $M=8$ seems to be the dimension of diminishing returns for the rolling QR method for this problem: there is no significant difference between the times and speedups attained for $M=8$ compared with $M=12$; if anything, $M=12$ yields a method that is slightly slower.

The real winners here, however, are the extrapolation methods, which attain a speedup of $70 \%$ or greater in most cases and up to $85 \%$ with some configurations. This improved time to solution comes in spite of the fact that the projection methods, exemplified by the rolling QR scheme, attain lower solver iteration counts, in particular for the pressure problem. The average PCG iteration counts per time step required for the velocity and pressure solves are presented in Tables 3 and 4, respectively.

As an example, Table 4, shows that for $\mathrm{Re}=200, \mathrm{QR}(8)$ performs roughly $15 \%$ fewer iterations than $\operatorname{EXTRAP}(2,8)$ when solving the pressure problem, yet the latter is actually about $10 \%$ faster according to Table 2 . Part of this is due to the fact that $\operatorname{EXTRAP}(2,8)$ performs about $5 \%$ fewer iterations on average than $\mathrm{QR}(8)$ for the velocity problems, but it is also due to the fact that $\operatorname{EXTRAP}(2,8)$ does not have to perform the (relatively) expensive update step that $\mathrm{QR}(8)$ does.

We observe that, in general, the extrapolation methods tend to yield slightly lower iteration counts than the projection methods for the velocity problems, while for the pressure problems, the opposite is true. The reason for this difference is not immediately clear. Note that reducing pressure iterations is in general more valuable than reducing velocity iterations, as a pressure iteration is considerably more expensive to effect than a velocity iteration owing to the more sophisticated preconditioner.

Focusing on the pressure solves, Figure 3 a shows the number of pressure iterations taken at several time steps during the $\mathrm{Re}=500$ run for the various strategies, using a space of dimension $M=8$. The projection methods begin to produce substantially lower iteration counts versus using the solution at the last time step after about 10 time steps, while the extrapolation methods take closer to 20 time steps to do the same. The plot shows the stark difference between the classic and rolling QR schemes: every 8 steps, the iteration count for the classic scheme jumps to match that for using the previous time step's solution, reflecting the fact that it periodically discards the history space. In contrast, the rolling QR scheme maintains counts that are consistently low. After about 45 time steps, the iteration counts for $\operatorname{EXTRAP}(4,8)$ 


\begin{tabular}{cl|cc|cc|cc}
\hline \multicolumn{2}{c}{ Method } & & \multicolumn{2}{c}{$\mathrm{Re}=200$} & \multicolumn{2}{c}{$\mathrm{Re}=500$} & \multicolumn{2}{c}{$\mathrm{Re}=1000$} \\
\hline Last time step & & 2629 & $(1.00)$ & 2586 & $(1.00)$ & 2568 & $(1.00)$ \\
\hline & $M=4$ & 1872 & $(1.40)$ & 1875 & $(1.38)$ & 1901 & $(1.35)$ \\
Classic & $M=8$ & 1721 & $(1.53)$ & 1712 & $(1.51)$ & 1748 & $(1.47)$ \\
& $M=12$ & 1673 & $(1.57)$ & 1666 & $(1.55)$ & 1701 & $(1.51)$ \\
\hline & $M=4$ & 1620 & $(1.62)$ & 1643 & $(1.57)$ & 1651 & $(1.56)$ \\
Rolling QR & $M=8$ & 1589 & $(1.65)$ & 1591 & $(1.63)$ & 1644 & $(1.56)$ \\
& $M=12$ & 1625 & $(1.62)$ & 1637 & $(1.58)$ & 1677 & $(1.53)$ \\
\hline \multirow{2}{*}{ Extrapolation } & $M=4$ & 1527 & $(1.72)$ & 1519 & $(1.70)$ & 1538 & $(1.67)$ \\
(Least-sq., $m=2)$ & $M=8$ & 1432 & $(1.84)$ & 1434 & $(1.80)$ & 1459 & $(1.76)$ \\
& $M=12$ & 1418 & $(1.85)$ & 1421 & $(1.82)$ & 1460 & $(1.76)$ \\
\hline \multirow{2}{*}{ Extrapolation } & $M=4$ & 1692 & $(1.55)$ & 1683 & $(1.54)$ & 1677 & $(1.53)$ \\
(Least-sq., $m=3)$ & $M=8$ & 1478 & $(1.78)$ & 1475 & $(1.75)$ & 1475 & $(1.74)$ \\
& $M=12$ & 1414 & $(1.86)$ & 1408 & $(1.84)$ & 1411 & $(1.82)$ \\
\hline \multirow{2}{*}{ Extrapolation } & $M=4$ & - & - & - & - & - & - \\
(Least-sq., $m=4)$ & $M=8$ & 1589 & $(1.65)$ & 1577 & $(1.64)$ & 1570 & $(1.64)$ \\
& $M=12$ & 1474 & $(1.78)$ & 1464 & $(1.77)$ & 1468 & $(1.75)$ \\
\hline \multirow{2}{*}{ Extrapolation } & $M=4$ & 1531 & $(1.72)$ & 1524 & $(1.70)$ & 1540 & $(1.67)$ \\
(Sparse, $m=2)$ & $M=8$ & 1420 & $(1.85)$ & 1424 & $(1.82)$ & 1448 & $(1.77)$ \\
& $M=12$ & 1405 & $(1.87)$ & 1413 & $(1.83)$ & 1440 & $(1.78)$ \\
\hline \multirow{2}{*}{ Extrapolation } & $M=4$ & 1693 & $(1.55)$ & 1682 & $(1.54)$ & 1677 & $(1.53)$ \\
(Sparse, $m=3)$ & $M=8$ & 1492 & $(1.76)$ & 1483 & $(1.74)$ & 1483 & $(1.73)$ \\
& $M=12$ & 1416 & $(1.86)$ & 1412 & $(1.83)$ & 1418 & $(1.81)$ \\
\hline \multirow{2}{*}{ Extrapolation } & $M=4$ & - & - & - & - & - & - \\
(Sparse, $m=4)$ & $M=8$ & 1610 & $(1.63)$ & 1595 & $(1.62)$ & 1594 & $(1.61)$ \\
& $M=12$ & 1489 & $(1.77)$ & 1476 & $(1.75)$ & 1481 & $(1.73)$ \\
\hline
\end{tabular}

Table 2: Simulation times (in seconds) for the 3D fence flow test problem to a final time of $t=1.00$ (approximately 8700 time steps) for each of the initial guess strategies. The parameter $M$ is the maximum dimension of the history space used by the methods. The numbers in parentheses give the speedups attained by the methods relative to the strategy of using the solution at the last time step.

reach the same level as rolling QR. The counts for $\operatorname{EXTRAP}(2,8)$ and $\operatorname{EXTRAP}(3,8)$ are somewhat higher but remain on a downward trend.

Figure $3 \mathrm{~b}$ illustrates the long-term behavior of the pressure solver iteration counts over the course of the full simulation, displaying the average at each time step of the iteration counts over all the time steps taken to that point. While all of the strategies for selecting initial guesses result in iteration counts that trend downward with time, the projection and extrapolation methods yield average iteration counts that are considerably lower. After 8700 time steps, pressure solves seeded with the previous time step's solution as the initial guess take about 15 PCG iterations on average. For the classic projection scheme with $M=8$, the long-term average is just under 5 iterations per solve. For the rolling QR scheme with $M=8$, it is about 


\begin{tabular}{cl|cc|cc|cc}
\hline \multicolumn{2}{c}{ Method } & & \multicolumn{2}{c|}{$\mathrm{Re}=200$} & \multicolumn{2}{c|}{$\mathrm{Re}=500$} & \multicolumn{2}{c}{$\mathrm{Re}=1000$} \\
\hline Last time step & 26.00 & $(1.00)$ & 19.68 & $(1.00)$ & 15.36 & $(1.00)$ \\
\hline & $M=4$ & 10.71 & $(2.43)$ & 8.63 & $(2.28)$ & 7.96 & $(1.93)$ \\
Classic & $M=8$ & 7.61 & $(3.41)$ & 5.86 & $(3.36)$ & 5.52 & $(2.78)$ \\
& $M=12$ & 6.40 & $(4.06)$ & 4.94 & $(3.98)$ & 4.70 & $(3.26)$ \\
\hline \multirow{2}{*}{ Rolling QR } & $M=4$ & 5.23 & $(4.97)$ & 4.46 & $(4.42)$ & 3.82 & $(4.02)$ \\
& $M=8$ & 6.15 & $(4.23)$ & 5.59 & $(3.52)$ & 4.91 & $(3.13)$ \\
& $M=12$ & 6.04 & $(4.30)$ & 5.61 & $(3.51)$ & 5.16 & $(2.98)$ \\
\hline \multirow{2}{*}{ Extrapolation } & $M=4$ & 5.14 & $(5.06)$ & 4.49 & $(4.38)$ & 5.49 & $(2.80)$ \\
(Least-sq., $m=2)$ & $M=8$ & 5.81 & $(4.48)$ & 6.15 & $(3.20)$ & 6.46 & $(2.38)$ \\
& $M=12$ & 6.81 & $(3.82)$ & 6.79 & $(2.90)$ & 6.95 & $(2.21)$ \\
\hline \multirow{2}{*}{ Extrapolation } & $M=4$ & 5.56 & $(4.67)$ & 4.68 & $(4.20)$ & 3.92 & $(3.92)$ \\
(Least-sq., $m=3)$ & $M=8$ & 4.07 & $(6.39)$ & 3.74 & $(5.26)$ & 3.34 & $(4.60)$ \\
& $M=12$ & 3.76 & $(6.91)$ & 3.55 & $(5.55)$ & 3.54 & $(4.34)$ \\
\hline \multirow{2}{*}{ Extrapolation } & $M=4$ & - & - & - & - & - & - \\
(Least-sq., $m=4)$ & $M=8$ & 5.05 & $(5.14)$ & 4.10 & $(4.79)$ & 3.15 & $(4.88)$ \\
& $M=12$ & 3.92 & $(6.63)$ & 3.19 & $(6.17)$ & 3.21 & $(4.78)$ \\
\hline \multirow{2}{*}{ Extrapolation } & $M=4$ & 5.13 & $(5.07)$ & 4.76 & $(4.13)$ & 5.62 & $(2.73)$ \\
(Sparse, $m=2)$ & $M=8$ & 5.23 & $(4.97)$ & 5.85 & $(3.36)$ & 6.40 & $(2.40)$ \\
& $M=12$ & 6.07 & $(4.28)$ & 6.49 & $(3.03)$ & 6.73 & $(2.28)$ \\
\hline \multirow{2}{*}{ Extrapolation } & $M=4$ & 5.55 & $(4.68)$ & 4.65 & $(4.23)$ & 3.92 & $(3.92)$ \\
(Sparse, $m=3)$ & $M=8$ & 4.22 & $(6.17)$ & 3.52 & $(5.59)$ & 3.32 & $(4.62)$ \\
& $M=12$ & 3.80 & $(6.84)$ & 3.37 & $(5.84)$ & 3.43 & $(4.47)$ \\
\hline \multirow{2}{*}{ Extrapolation } & $M=4$ & - & - & - & - & - & - \\
(Sparse, $m=4)$ & $M=8$ & 4.98 & $(5.22)$ & 3.72 & $(5.30)$ & 3.16 & $(4.86)$ \\
& $M=12$ & 4.42 & $(5.89)$ & 3.25 & $(6.06)$ & 3.21 & $(4.79)$ \\
\hline
\end{tabular}

Table 3: Average number of velocity solver PCG iterations at each time step for the same problem as in Table 2. The numbers in parentheses give the factor by which the various methods reduce the number of iterations relative to using the solution at the last time step as an initial guess.

2.5. $\operatorname{EXTRAP}(2,8)$ and $\operatorname{EXTRAP}(3,8)$ compete closely with $\mathrm{QR}(8)$, with long-term averages of about 2.6 and 3.5 , respectively.

The performance of the sparse extrapolation methods is nearly indistinguishable from that of the least-squares methods, though the pressure iteration counts for the sparse methods are, in general, very slightly higher. This validates our claim made in Section 3.3 that even though the vector of extrapolation coefficients for the sparse method does not satisfy a minimality property like that for the least-squares method, the sparse method is still a viable approach.

6.4. Effect of the Stopping Criterion. The results just reported depend to some extent on the stopping criterion used for the Krylov solver. In libParanumal, 


\begin{tabular}{cl|cc|cc|cc}
\hline \multicolumn{2}{c}{ Method } & & \multicolumn{2}{c|}{$\mathrm{Re}=200$} & \multicolumn{2}{c|}{$\mathrm{Re}=500$} & \multicolumn{2}{c}{$\mathrm{Re}=1000$} \\
\hline Last time step & 14.77 & $(1.00)$ & 15.19 & $(1.00)$ & 15.60 & $(1.00)$ \\
\hline & $M=4$ & 6.57 & $(2.25)$ & 6.91 & $(2.20)$ & 7.28 & $(2.14)$ \\
Classic & $M=8$ & 4.67 & $(3.16)$ & 4.81 & $(3.16)$ & 5.26 & $(2.97)$ \\
& $M=12$ & 3.81 & $(3.87)$ & 3.92 & $(3.87)$ & 4.35 & $(3.59)$ \\
\hline & $M=4$ & 3.60 & $(4.11)$ & 3.96 & $(3.84)$ & 4.07 & $(3.83)$ \\
Rolling QR & $M=8$ & 2.31 & $(6.40)$ & 2.36 & $(6.44)$ & 3.03 & $(5.16)$ \\
& $M=12$ & 1.83 & $(8.06)$ & 2.02 & $(7.53)$ & 2.50 & $(6.25)$ \\
\hline \multirow{2}{*}{ Extrapolation } & $M=4$ & 4.07 & $(3.63)$ & 4.05 & $(3.75)$ & 4.08 & $(3.83)$ \\
(Least-sq., $m=2)$ & $M=8$ & 2.70 & $(5.47)$ & 2.64 & $(5.75)$ & 2.86 & $(5.45)$ \\
& $M=12$ & 2.28 & $(6.47)$ & 2.29 & $(6.63)$ & 2.71 & $(5.75)$ \\
\hline \multirow{2}{*}{ Extrapolation } & $M=4$ & 6.07 & $(2.43)$ & 6.07 & $(2.50)$ & 6.05 & $(2.58)$ \\
(Least-sq., $m=3)$ & $M=8$ & 3.55 & $(4.16)$ & 3.54 & $(4.29)$ & 3.55 & $(4.40)$ \\
& $M=12$ & 2.71 & $(5.46)$ & 2.65 & $(5.74)$ & 2.63 & $(5.94)$ \\
\hline \multirow{2}{*}{ Extrapolation } & $M=4$ & - & - & - & - & - & - \\
(Least-sq., $m=4)$ & $M=8$ & 4.78 & $(3.09)$ & 4.76 & $(3.19)$ & 4.77 & $(3.27)$ \\
& $M=12$ & 3.44 & $(4.30)$ & 3.40 & $(4.46)$ & 3.40 & $(4.59)$ \\
\hline \multirow{2}{*}{ Extrapolation } & $M=4$ & 4.14 & $(3.57)$ & 4.09 & $(3.72)$ & 4.10 & $(3.81)$ \\
(Sparse, $m=2)$ & $M=8$ & 2.74 & $(5.40)$ & 2.67 & $(5.69)$ & 2.82 & $(5.52)$ \\
& $M=12$ & 2.40 & $(6.15)$ & 2.42 & $(6.27)$ & 2.67 & $(5.84)$ \\
\hline \multirow{2}{*}{ Extrapolation } & $M=4$ & 6.08 & $(2.43)$ & 6.05 & $(2.51)$ & 6.06 & $(2.58)$ \\
(Sparse, $m=3)$ & $M=8$ & 3.77 & $(3.92)$ & 3.75 & $(4.05)$ & 3.73 & $(4.19)$ \\
& $M=12$ & 2.87 & $(5.14)$ & 2.87 & $(5.29)$ & 2.89 & $(5.40)$ \\
\hline \multirow{2}{*}{ Extrapolation } & $M=4$ & - & - & - & - & - & - \\
(Sparse, $m=4)$ & $M=8$ & 5.12 & $(2.89)$ & 5.10 & $(2.98)$ & 5.12 & $(3.05)$ \\
& $M=12$ & 3.68 & $(4.01)$ & 3.68 & $(4.13)$ & 3.69 & $(4.23)$ \\
\hline
\end{tabular}

Table 4: Average number of pressure solver PCG iterations at each time step for the same problem as in Table 2. The numbers in parentheses give the factor by which the various methods reduce the number of iterations relative to using the solution at the last time step as an initial guess.

the default is to stop the iteration when

$$
\|r\|_{2}<\varepsilon \max \left(\left\|r_{0}\right\|_{2}, 1\right),
$$

where $r$ is the current residual, $r_{0}$ is the initial residual, and $\varepsilon$ is a chosen tolerance. By default, libParanumal uses $\varepsilon=10^{-8}$. This criterion stops iterating when the residual has been reduced to $\varepsilon$ in a relative sense when the initial residual is large and in an absolute sense when the initial residual is small. The results of the previous section were found using this default criterion.

One alternative to this criterion would be to demand

$$
\|r\|_{2}<\varepsilon \max \left(\|b\|_{2}, 1\right),
$$

i.e., to ask for a reduction relative not to the size of the initial residual but to the size 


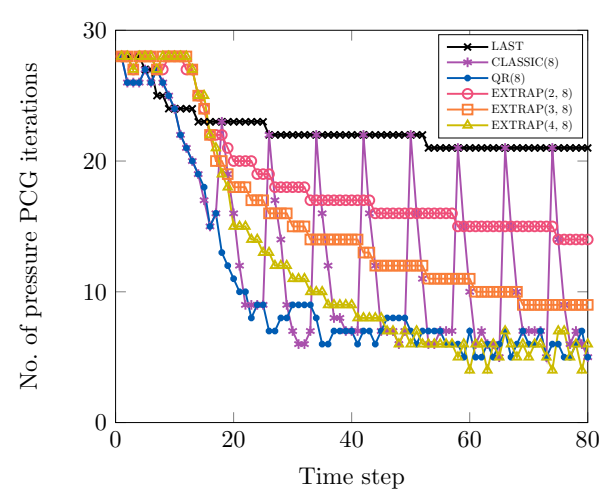

(a)

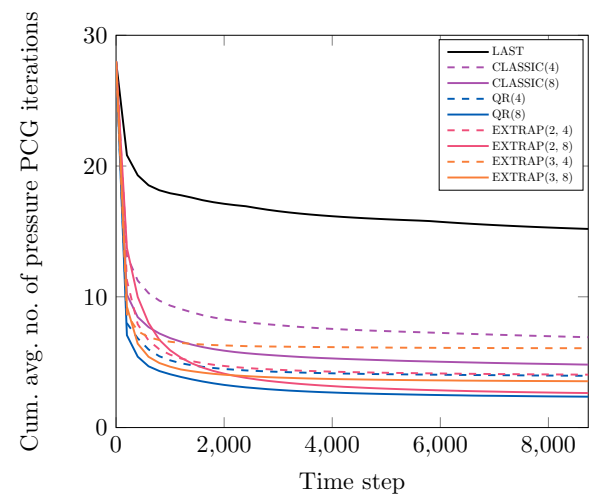

(b)

Fig. 3: (a) Pressure solver PCG iteration counts at the first 80 time steps for the 3D fence flow test problem at $R e=500$ for several initial guess strategies. (b) Cumulative average pressure iteration counts over the entire duration of the same simulation to a final time of $t=1.00$ (approximately 8700 time steps).

of the right-hand side $b$. The main difference between (6.1) and (6.2) is that if the initial guess is good $\left(\left\|r_{0}\right\|_{2}\right.$ is small), the former criterion will almost always collapse to $\|r\|_{2}<\varepsilon$, while the latter will typically be $\|r\|_{2}<\varepsilon\|b\|_{2}$, as $\|b\|_{2}>1$ in most cases in the flow simulations we consider. That means (6.2) can be a less stringent requirement than (6.1) with a good initial guess.

We shall not attempt to determine which of the two criteria is "superior," as this depends on one's accuracy requirements. Instead, let us examine the effect of the choice of criterion on the efficacy of the initial guess methods. Table 5 shows the times to solution for our test problem with $R e=500$ for each of the initial guess methods for both stopping criteria with $\varepsilon=10^{-8}$. As the table shows, criterion (6.2) results in shorter solution times, and the more sophisticated initial guess methods at less effective at reducing the time compared to running under criterion (6.1). Still, even under (6.2), the extrapolation methods are able to attain speedups comparable to (and even slightly better than) those attained by the projection methods.

Unless otherwise indicated, we will continue to use criterion (6.1) with $\varepsilon=10^{-8}$ in what follows.

6.5. Comparison with Naive Extrapolation. It is worth dwelling briefly on how the stabilized extrapolation methods we have proposed compare with naive extrapolation via Lagrange interpolation as described in Section 3.1. As we wrote above, this method is known to perform poorly as the polynomial degree increases. This is confirmed by the numbers in Table 6, which show a steady degradation in both solution time and pressure solver iterations when applied to our test problem with $\operatorname{Re}=500$. Comparing with Tables 2 and 4 , we see, for instance, that $\operatorname{EXTRAP}(5,6)$ is clearly inferior to $\operatorname{EXTRAP}(2,4)$, resulting in more than double the number of pressure iterations per time step on average despite a $50 \%$ larger history space.

The reason for this poor performance is related to the well-understood bad behavior of polynomial interpolation in equi-spaced points. We can quantify this poor behavior by introducing the notion of a Lebesgue constant for the extrapolation schemes, 
A. P. AUSTIN, N. CHALMERS, AND T. WARBURTON

\begin{tabular}{cl|ll|ll}
\hline \multicolumn{2}{c}{ Method } & & \multicolumn{2}{c}{ Criterion (6.1) } & \multicolumn{2}{c}{ Criterion (6.2) } \\
\hline Last time step & 2586 & $(1.00)$ & 1990 & $(1.00)$ \\
\hline \multirow{2}{*}{ Classic } & $M=4$ & 1875 & $(1.38)$ & 1791 & $(1.11)$ \\
& $M=8$ & 1712 & $(1.51)$ & 1676 & $(1.19)$ \\
& $M=12$ & 1666 & $(1.55)$ & 1651 & $(1.21)$ \\
\hline \multirow{3}{*}{ Rolling QR } & $M=4$ & 1643 & $(1.57)$ & 1526 & $(1.30)$ \\
& $M=8$ & 1591 & $(1.63)$ & 1570 & $(1.27)$ \\
& $M=12$ & 1637 & $(1.58)$ & 1658 & $(1.20)$ \\
\hline \multirow{2}{*}{ Extrapolation } & $M=4$ & 1519 & $(1.70)$ & 1496 & $(1.33)$ \\
(Least-sq., $m=2)$ & $M=8$ & 1434 & $(1.80)$ & 1413 & $(1.41)$ \\
& $M=12$ & 1421 & $(1.82)$ & 1355 & $(1.47)$ \\
\hline \multirow{2}{*}{ Extrapolation } & $M=4$ & 1683 & $(1.54)$ & 1909 & $(1.04)$ \\
(Least-sq., $m=3)$ & $M=8$ & 1475 & $(1.75)$ & 1467 & $(1.36)$ \\
& $M=12$ & 1408 & $(1.84)$ & 1398 & $(1.42)$ \\
\hline \multirow{2}{*}{ Extrapolation } & $M=4$ & - & - & - & - \\
(Least-sq., $m=4)$ & $M=8$ & 1577 & $(1.64)$ & 1582 & $(1.26)$ \\
& $M=12$ & 1464 & $(1.77)$ & 1464 & $(1.36)$ \\
\hline
\end{tabular}

Table 5: Simulation times (in seconds) for the 3D fence flow test problem at $\mathrm{Re}=500$ to a final time of $t=1.00$ (approximately 8700 time steps) for each of the initial guess strategies using the two stopping criteria (6.1) and (6.2) with $\varepsilon=10^{-8}$. The numbers in parentheses give the speedups attained by the methods relative to the strategy of using the solution at the last time step.

\begin{tabular}{ccccc}
\hline Method & \multicolumn{2}{c}{ Solution time } & \multicolumn{2}{c}{ Pressure iters. } \\
\hline Last time step & 2586 & $(1.00)$ & 15.19 & $(1.00)$ \\
\hline EXTRAP $(2,3)$ & 1589 & $(1.63)$ & 4.98 & $(3.05)$ \\
EXTRAP $(3,4)$ & 1683 & $(1.54)$ & 6.07 & $(2.50)$ \\
EXTRAP $(4,5)$ & 1795 & $(1.44)$ & 7.34 & $(2.07)$ \\
EXTRAP $(5,6)$ & 1905 & $(1.36)$ & 8.62 & $(1.76)$ \\
\hline
\end{tabular}

Table 6: Solution times and average pressure solver iteration counts for each of four extrapolation methods based on naive polynomial interpolation applied to the fence flow test problem with $\mathrm{Re}=500$. The numbers in parentheses give the improvements attained by the methods relative to the strategy of using the solution at the last time step.

which is just a suitable norm of the linear operator that maps the history data to the extrapolated value. Conceptually, the Lebesgue constant for a scheme measures the extent to which it amplifies perturbations to the history data, such as those resulting from round-off error or the fact that the linear systems are solved only as accurately as demanded by the Krylov solver tolerance.

In our case, the appropriate definition for the Lebesgue constant $\Lambda$ associated to 


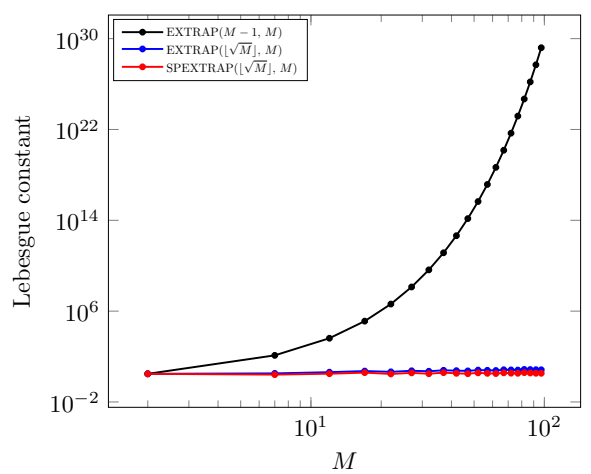

(a)

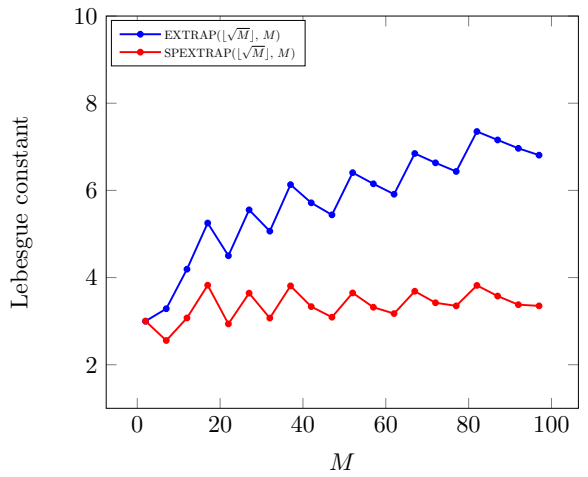

(b)

Fig. 4: (a) Lebesgue constants as a function of history space dimension $M$ for the naive extrapolation scheme, a stable extrapolation scheme, and its sparse counterpart. Note the logarithmic scaling of the axes. (b) Same plot on linear axes, omitting the data for the naive extrapolation scheme.

the extrapolation scheme with coefficient vector $\beta^{T}$ is

$$
\Lambda=\max _{\|x\|_{\infty}=1}\left|\beta^{T} x\right|=\|\beta\|_{1},
$$

i.e., the sum of the absolute values of the extrapolation coefficients. The following theorem is easy to prove:

THEOREM 6.1. The Lebesgue constant for the naive $\operatorname{EXTRAP}(M-1, M)$ extrapolation scheme is $\Lambda=2^{M}-1$.

Proof. By (3.2),

$$
\|\beta\|_{1}=\sum_{i=1}^{M}\left(\begin{array}{c}
M \\
i-1
\end{array}\right)=2^{M}-1 .
$$

This explosive exponential growth of the Lebesgue constant is what ultimately renders the naive extrapolation scheme useless in practice. As Table 6 illustrates, $M$ does not even need to be very large before the negative impacts are felt.

The Lebesgue constants for the stabilized schemes do not have convenient closed forms, but we can compute them easily. Figure 4 plots the Lebesgue constants as a function of $M$ for the native scheme, the stable scheme $\operatorname{EXTRAP}(\lfloor\sqrt{M}\rfloor, M)$, and the corresponding sparse stable scheme, where $\lfloor\cdot\rfloor$ denotes the "floor" (greatest integer) function. The Lebesgue constants for the stabilized methods do grow but at a considerably slower rate than does the Lebesgue constant for the naive scheme.

6.6. Reynolds Number Dependence. Table 2 suggests that the effectiveness of the initial guess methods degrades at least mildly as the Reynolds number increases. To investigate this, we ran our solver on our 3D fence flow test problem for several flows with Reynolds numbers up to $\mathrm{Re}=7,000$ and measured the average number of iterations taken by the pressure solver. The results are displayed in Figure 5. We observe that, indeed, the iteration counts do rise for both methods as the Reynolds number increases. 


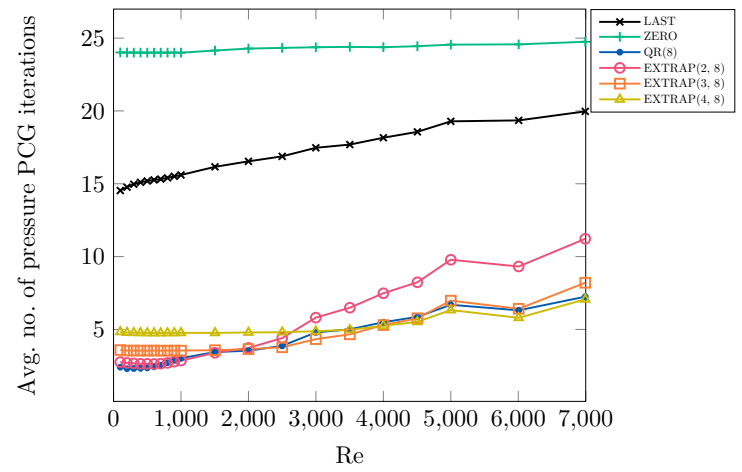

Fig. 5: Average number of pressure solver PCG iterations for the 3D fence flow test problem, run to a final time of $t=1.00$ (approximately 8700 time steps) for flows with Reynolds numbers varying from 200 to 7,000 for several initial guess strategies. Legend labels correspond to Table 1. "ZERO" gives the results obtained using a zero initial guess for the pressure solution at each time step.

We also ran simulations at the same Reynolds numbers that used a zero initial guess at all time steps. The average pressure iteration counts for these simulations are nearly steady at approximately 24 PCG steps per solve, irrespective of Reynolds number. This shows that our preconditioner is Re-independent (or at most only very weakly Re-dependent) and hence that Re dependence of the iteration counts of the initial guess methods is due to the methods themselves and how they interact with the equations (5.1) and our discretization, not our preconditioner.

At $\operatorname{Re}=7,000$, the effectiveness of the initial guess strategy of using the previous time step's solution is questionable, as it saves just 4 iterations per solve on average compared with using a zero initial guess. While the projection and extrapolation schemes' effectiveness at $\mathrm{Re}=7,000$ is diminished compared with low Reynolds numbers, they still take fewer than half the number of iterations as a zero initial guess.

6.7. Using Different Methods for Velocity and Pressure. The experiments reported in the previous sections all use the same initial guess scheme for both the velocity and the pressure solves. In fact, it may be advantageous to use different schemes for each. Table 7 shows the results obtained on our test problem with $\operatorname{Re}=500$ using the $\operatorname{EXTRAP}(3,8)$ scheme for velocity and several different schemes for pressure except for the first row, which gives the results for using the solution at the last time step as the initial guess for both problems.

Comparing with Table 2, we see that when the initial guesses for pressure are found using an extrapolation scheme, using $\operatorname{EXTRAP}(3,8)$ for velocity leads to essentially the same results as using the same extrapolation scheme for velocity as for pressure. On the other hand, using $\operatorname{EXTRAP}(3,8)$ for velocity and the rolling QR projection scheme for pressure is a marked improvement over using the rolling QR scheme for both. The reason is that the extrapolation scheme produces lower velocity iteration counts, cf. Table 3. Despite this improvement, however, schemes that use extrapolation for both velocity and pressure still attain slightly better times.

Table 7 shows only a small subset of the possible combinations. We leave the determination of the "best" combination as a matter for future work. 


\begin{tabular}{cl|cc|cc|cc}
\hline \multicolumn{2}{c|}{ Method } & \multicolumn{2}{|c|}{ Solution time } & \multicolumn{3}{c|}{ Velocity iters. } & \multicolumn{2}{c}{ Pressure iters. } \\
\hline \multicolumn{2}{c|}{ Last time step } & 2586 & $(1.00)$ & 19.68 & $(1.00)$ & 15.19 & $(1.00)$ \\
\hline \multirow{2}{*}{ Rolling QR } & $M=4$ & 1540 & $(1.68)$ & 3.83 & $(5.14)$ & 3.93 & $(3.86)$ \\
& $M=8$ & 1442 & $(1.79)$ & 3.85 & $(5.11)$ & 2.39 & $(6.36)$ \\
& $M=12$ & 1437 & $(1.80)$ & 3.78 & $(5.20)$ & 2.06 & $(7.36)$ \\
\hline \multirow{2}{*}{ Extrapolation } & $M=4$ & 1512 & $(1.71)$ & 3.75 & $(5.25)$ & 4.02 & $(3.78)$ \\
(Least-sq., $m=2)$ & $M=8$ & 1408 & $(1.84)$ & 3.83 & $(5.14)$ & 2.68 & $(5.68)$ \\
& $M=12$ & 1381 & $(1.87)$ & 3.80 & $(5.18)$ & 2.32 & $(6.54)$ \\
\hline \multirow{2}{*}{ Extrapolation } & $M=4$ & 1672 & $(1.55)$ & 3.77 & $(5.22)$ & 6.01 & $(2.53)$ \\
(Least-sq., $m=3)$ & $M=8$ & 1476 & $(1.75)$ & 3.74 & $(5.26)$ & 3.54 & $(4.29)$ \\
& $M=12$ & 1406 & $(1.84)$ & 3.77 & $(5.21)$ & 2.64 & $(5.76)$ \\
\hline \multirow{2}{*}{ Extrapolation } & $M=4$ & - & - & - & - & - & - \\
(Least-sq., $m=4)$ & $M=8$ & 1571 & $(1.65)$ & 3.76 & $(5.23)$ & 4.73 & $(3.21)$ \\
& $M=12$ & 1467 & $(1.76)$ & 3.82 & $(5.16)$ & 3.40 & $(4.47)$ \\
\hline
\end{tabular}

Table 7: Simulation times (in seconds) for the 3D fence flow test problem to a final time of $t=1.00$ (approximately 8700 time steps) using $\operatorname{EXTRAP}(3,8)$ for velocity and the listed initial guess strategies for pressure except for the first row, which gives the results for using the solution at the last time step as the initial guess for both velocity and pressure. The numbers in parentheses give the improvements attained by the methods relative to the first row.

7. Conclusion. We have studied the effectiveness of projection and extrapolation methods for computing initial guesses for the solutions to linear systems that arise within numerical PDE solvers. We have proposed stabilized extrapolation techniques based on least-squares polynomial approximation and polynomial interpolation in well-chosen (non-equi-spaced) points and shown that both they and projection techniques can be implemented efficiently on GPUs. In spite of their simplicity, we have found the proposed extrapolation methods to perform comparably to - and, in some cases, slightly better than - equivalent projection methods while requiring less storage, moving less data, and performing fewer operations that require global communication. In future work, we plan to study the impact of these techniques on the scalability of GPU-accelerated PDE solvers across multiple GPUs and compute nodes.

Acknowledgments. We acknowledge Advanced Research Computing at Virginia Tech for providing the computational resources with which we conducted the bulk of our experiments.

\section{Appendix A. Details of Data Operation Counts.}

In this section, we provide details of the reasoning used to arrive at the data movement operation counts in Table 1. For the projection methods, the reader may find it helpful to refer to Algorithms 2.1 and 2.2. All vectors are of length $N_{\text {total }}$. As discussed above, we assume that $N_{\text {total }} \gg M$ so that terms involving only $M$ can be neglected.

First, we note the following basic counts:

- Taking a linear combination of $M$ vectors requires loading $M N_{\text {total }}$ vector entries and storing $N_{\text {total }}$, for a total of $(M+1) N_{\text {total }}$ operations. 
- If, at the same time, we add that linear combination to a given vector, then we must also load that vector, bringing the count to $(M+2) N_{\text {total }}$.

- Computing the inner products of $M$ vectors with a given vector requires loading $(M+1) N_{\text {total }}$ values and storing the $M$ inner products. Neglecting the latter, we have $(M+1) N_{\text {total }}$ operations.

- Applying the Laplacian operator in our matrix-free implementation requires loading the input and storing the output ( $2 N_{\text {total }}$ values moved) and loading the geometric factors $\left(7 N_{\text {total }}\right.$ values moved in $\left.3 \mathrm{D}\right)$. In all, this comes to $9 N_{\text {total }}$ data movement operations.

A.1. Forming the Initial Guess. The projection methods form their initial guesses by taking the inner product of the new right-hand side with the vectors in the right-hand side history space. With a space of dimension $M$, this comes to $(M+1) N_{\text {total }}$ operations. They then take the computed inner products and use them for coefficients in a linear combination of $M$ solution vectors. That takes another $(M+1) N_{\text {total }}$ operations for a total of $2(M+1) N_{\text {total }}$. Once the history space is full, $\mathrm{QR}(M)$ will use this many operations every time, but CLASSIC $(M)$ doesn't always use a space of size exactly $M$ : it uses spaces of size $1, \ldots, M$ in a cycle. Averaging the data movement operations involved in forming the initial guess over these sizes, we obtain a count $(M+3) N_{\text {total }}$ for $\operatorname{CLASSIC}(M)$.

$\operatorname{EXTRAP}(m, M)$ forms the initial guess from a linear combination of $M$ vectors for a total operation count of $(M+1) N_{\text {total }}$. $\operatorname{SPEXTRAP}(m, M)$ does the same but uses only $m+1$ vectors.

A.2. Updating the History Space. The projection methods update their spaces using twice-iterated Gram-Schmidt. One round of Gram-Schmidt first computes the inner products of the $M$ vectors against the new vector, taking $(M+1) N_{\text {total }}$ operations. It then uses those inner products to subtract a linear combination of the $M$ vectors from the new vector, taking an additional $(M+2) N_{\text {total }}$ operations. The orthogonalization is only done to the right-hand side space, but the same linear combination must be carried out with the corresponding vectors in the solution history space. That takes another $(M+2) N_{\text {total }}$ operations, for a total of $(3 M+5) N_{\text {total }}$.

Twice-iterated Gram-Schmidt therefore takes $(6 M+10) N_{\text {total }}$ operations. Prior to running Gram-Schmidt, the projection operations apply the operator once, which takes $9 N_{\text {total }}$ operations. To finish the update, we have to compute the norm of the newly projected right-hand side vector, which performs one more load of length $N_{\text {total }}$. We then scale the new right-hand side and corresponding solution vectors by this norm and store them in the history arrays, which takes another $4 N_{\text {total }}$ movement operations (load and store two vectors), ignoring the need to load the norm itself. Thus, the total amount of data moved during this part of the update process is $(6 M+24) N_{\text {total }}+6 M$. To obtain the count for CLASSIC $(M)$, we simply average this count over the restart cycle like we did before, giving $(3 M+27) N_{\text {total }}$.

In addition to the twice-iterated Gram-Schmidt update, $\mathrm{QR}(M)$ must do the QR update at every time step. This requires loading the $M \times M$ matrix $R$, which we neglect. Then, we apply a sequence of $M-1$ Givens rotations, each of which operates on a pair of columns from the right-hand side history matrix. It would seem that each rotation requires $4 N_{\text {total }}$ data movement operations: load the two columns to be processed and then store the results, but by being keeping the column in common used by two successive rotations in registers, we can cut this in half to $2 N_{\text {total }}$ operations per rotation. We have to do the same operations on the solution space, which brings us back to $4 N_{\text {total }}$ operations per rotation, giving a total of $4(M-1) N_{\text {total }}$ operations 
for the whole sequence of Givens sweeps. Adding in the $2 N_{\text {total }}$ loads for the initial columns and the $2 N_{\text {total }}$ stores for zeroing out the final columns, we get a total of $4 M N_{\text {total }}$ operations. Adding the counts for the twice-iterated Gram-Schmidt and QR update operations together, $\mathrm{QR}(M)$ requires $(10 M+24) N_{\text {total }}+M^{2}+6 M$ data movement operations to update its solution space.

The extrapolation methods do not require any operations to update the history space, provided that the linear solver stores the solutions in place in the history array.

\section{REFERENCES}

[1] gslib, 2020, https://github.com/Nek5000/gslib. Release 1.07.

[2] M. Abramowitz and I. A. Stegun, Handbook of Mathematical Functions With Formulas, Graphs, and Mathematical Tables, vol. 55 of Applied Mathematics Series, U.S. Dept. of Commerce, National Bureau of Standards, 1968. Seventh printing, with corrections.

[3] M. al Sayed Ali And M. Sadkane, Improved predictor schemes for large systems of linear ODEs, Electron. Trans. Numer. Anal., 39 (2012), pp. 253-270.

[4] L. Bos, S. De Marchi, A. Sommariva, and M. Vianello, Computing multivariate Fekete and Leja points by numerical linear algebra, SIAM J. Numer. Anal., 48 (2010), pp. 1984-1999, https://doi.org/10.1137/090779024.

[5] J. P. Boyd AND F. XU, Divergence (Runge Phenomenon) for least-squares polynomial approximation on an equispaced grid and Mock-Chebyshev subset interpolation, Appl. Math. Comput., 210 (2009), pp. 158-168, https://doi.org/10.1016/j.amc.2008.12.087.

[6] R. Brower, T. Ivanenko, A. Levi, and K. Orginos, Chronological inversion method for the Dirac matrix in hybrid Monte Carlo, Nucl. Phys. B, 484 (1997), pp. 353-374, https: //doi.org/10.1016/s0550-3213(96)00579-2.

[7] N. Chalmers, A. Karakus, A. P. Austin, K. Swirydowicz, and T. Warburton, libParanumal: a performance portable high-order finite element library, 2020, https://doi.org/10. 5281/zenodo.4004744, https://github.com/paranumal/libparanumal. Release 0.3.1.

[8] N. Christensen, Efficient Projection Space Updates for the Approximation of Iterative Solutions to Linear Systems with Successive Right Hand Sides, M.S. thesis, University of Illinois at Urbana-Champaign, 2017, https://doi.org/2142/99410.

[9] M. Clemens, M. Wilke, R. Schuhmann, and T. Weiland, Subspace projection extrapolation scheme for transient field simulations, IEEE Trans. Magnetics, 40 (2004), pp. 934-937, https://doi.org/10.1109/tmag.2004.824583.

[10] M. Clemens, M. Wilke, and T. Weiland, Extrapolation strategies in numerical schemes for transient magnetic field simulations, IEEE Trans. Magnetics, 39 (2003), pp. 1171-1174, https://doi.org/10.1109/tmag.2003.810523.

[11] J. W. Daniel, W. B. GragG, L. Kaufman, and G. W. Stewart, Reorthogonalization and stable algorithms for updating the Gram-Schmidt QR factorization, Math. Comp., 30 (1976), pp. 772-795, https://doi.org/10.1090/S0025-5718-1976-0431641-8.

[12] L. Demanet And A. Townsend, Stable extrapolation of analytic functions, Found. Comput. Math., 19 (2018), pp. 297-331, https://doi.org/10.1007/s10208-018-9384-1.

[13] M. O. Deville, P. F. Fischer, And E. H. Mund, High-Order Methods for Incompressible Fluid Flow, Cambridge University Press, Cambridge, UK, 2002.

[14] P. Fischer, J. Lottes, D. Pointer, And A. Siegel, Petascale algorithms for reactor hydrodynamics, J. Phys.: Conf. Ser., 125 (2008), p. 012076, https://doi.org/10.1088/1742-6596/ $125 / 1 / 012076$.

[15] P. F. FISCHER, Projection techniques for iterative solution of $A x=b$ with successive right-hand sides, Comput. Methods Appl. Mech. Eng., 163 (1998), pp. 193-204, https://doi.org/10. 1016/s0045-7825(98)00012-7.

[16] R. Gandham, High Performance High-Order Numerical Methods: Applications in Ocean Modeling, Ph.D. thesis, Rice University, 2015.

[17] G. H. Golub and C. F. Van Loan, Matrix Computations, Johns Hopkins University Press, Baltimore, 4th ed., 2013.

[18] A. Greenbaum, Iterative Methods for Solving Linear Systems, SIAM, Philadelphia, 1997.

[19] L. Grinberg AND G. E. KARNiAdAKIS, Extrapolation-based acceleration of iterative solvers: Application to simulation of 3D flows, Commun. Comput. Phys., 9 (2011), pp. 607-626, https://doi.org/10.4208/cicp.301109.080410s.

[20] J. M. Herbert and M. Head-Gordon, Accelerated, energy-conserving Born-Oppenheimer molecular dynamics via Fock matrix extrapolation, Phys. Chem. Chem. Phys., 7 (2005), 
p. 3269, https://doi.org/10.1039/b509494a.

[21] M. R. Hestenes and E. Stiefel, Methods of conjugate gradients for solving linear systems, J. Res. Nat. Bur. Stand., 49 (1952), pp. 409-436, https://doi.org/10.6028/jres.049.044.

[22] D. C. Joyce, Survey of extrapolation processes in numerical analysis, SIAM Rev., 13 (1971), pp. 435-490, https://doi.org/10.1137/1013092.

[23] A. Karakus, N. Chalmers, K. Świrydowicz, and T. Warburton, A GPU accelerated discontinuous Galerkin incompressible flow solver, J. Comput. Phys., 390 (2019), pp. 380-404, https://doi.org/10.1016/j.jcp.2019.04.010.

[24] G. E. Karniadakis And S. Sherwin, Spectral/hp Element Methods for Computational Fluid Dynamics, Oxford University Press, Oxford, 2nd ed., 2005.

[25] B. N. Parlett, The Symmetric Eigenvalue Problem, SIAM, Philadelphia, 1998.

[26] G. Pitton and L. Heltai, Accelerating the iterative solution of convection-diffusion problems using singular value decomposition, Numer. Linear Algebra Appl., 26 (2018), pp. e2211 (1-24), https://doi.org/10.1002/nla.2211.

[27] R. B. Platte, L. N. Trefethen, and A. B. Kuijlaars, Impossibility of fast stable approximation of analytic functions from equispaced samples, SIAM Rev., 53 (2011), pp. 308-318, https://doi.org/10.1137/090774707.

[28] P. Pulay and G. Fogarasi, Fock matrix dynamics, Chem. Phys. Lett., 386 (2004), pp. 272278, https://doi.org/10.1016/j.cplett.2004.01.069.

[29] E. A. Rakhmanov, Bounds for polynomials with a unit discrete norm, Ann. of Math. (2), 165 (2007), pp. 55-88, https://doi.org/10.4007/annals.2007.165.55.

[30] Y. SAAD, Iterative Methods for Sparse Linear Systems, SIAM, Philadelphia, 2nd ed., 2003.

[31] Y. SaAd AND M. H. Schultz, GMRES: A generalized minimal residual algorithm for solving nonsymmetric linear systems, SIAM J. Sci. Stat. Comput., 7 (1986), pp. 856-869, https: //doi.org/10.1137/0907058.

[32] K. S. ShTEREv, Iterative process acceleration of calculation of unsteady, viscous, compressible, and heat-conductive gas flows, Int. J. Numer. Meth. Fluids, 77 (2015), pp. 108-122, https: //doi.org/10.1002/fld.3979.

[33] A. Skarlatos, M. Clemens, and T. Weiland, Start vector generation for implicit newmark time integration of the wave equation, IEEE Trans. Magnetics, 42 (2006), pp. 631-634, https://doi.org/10.1109/tmag.2006.872008.

[34] A. G. TiJhuis AND P. M. Zwamborn, Marching on in anything: Solving electromagnetic field equations with a varying physical parameter, in Ultra-Wideband, Short-Pulse Electromagnetics 5, P. D. Smith and S. R. Cloude, eds., Kluwer Academic, New York, 2002, pp. 655-662, https://doi.org/10.1007/0-306-47948-6_78.

[35] L. N. Trefethen, Approximation Theory and Approximation Practice, SIAM, Philadelphia, 2013.

[36] H. M. Tufo III, Algorithms for Large-Scale Parallel Simulation of Unsteady Incompressible Flows in Three-Dimensional Complex Geometries, Ph.D. thesis, Brown University, 1998. 